hep-ph/9803259

UM-TH-98-05

March 1998

\title{
Disrupting the one-loop renormalization group invariant $M / \alpha$ in supersymmetry
}

\author{
Graham D. Kribs \\ Randall Physics Laboratory, University of Michigan \\ Ann Arbor, MI 48109-1120 \\ kribs@feynman.physics.lsa.umich.edu
}

\begin{abstract}
It is well known that in low energy supersymmetry the ratio of the gaugino mass to the gauge coupling squared, $M / \alpha$, is renormalization group invariant to one-loop. We present a systematic analysis of the corrections to this ratio, including standard two-loop corrections from gauge and Yukawa couplings, corrections due to an additional $U(1)^{\prime}$ gaugino, threshold corrections, superoblique corrections, corrections due to extra matter, GUT and Planck scale corrections, and "corrections" from messenger sectors with supersymmetry breaking communicated via gauge-mediation. We show that many of these effects induce corrections at the level of a few to tens of percent, but some could give much larger corrections, drastically disrupting the renormalization group extrapolation of the ratio to higher scales. Our analysis is essentially model-independent, and therefore can be used to determine the ambiguities in extrapolating the ratio in any given model between the weak scale and higher scales.
\end{abstract}




\section{Introduction}

It is well known that any hope of stabilizing a hierarchy of scales requires supersymmetry [1]. The disparity between the weak scale and the grand unified theory (GUT) scale or the Planck scale (hereafter lumped together as the "high scale") is bridged in a softly broken supersymmetric standard model by rendering scalar mass renormalization to be at most logarithmically divergent. The canonical approach to connecting these disparate scales is through the renormalization group (RG), and there has been enormous effort in calculating and evaluating the RG equations analytically and numerically, e.g. Refs. [2, 3, 目, 5, 6, 7, 8, 9, 10, 11, 12]. Most of this effort has been directed towards either the consequences of a given high scale theory (be it a GUT, string theory, or other high scale proposal) on weak scale phenomenology, or the consequences of present (or proposed) weak scale measurements on high scale physics.

A general, softly broken, weak scale supersymmetric model has a large number of additional parameters beyond those of the standard model (SM) [1]. Most of the parameters are interconnected through the RG equations, and therefore successful extrapolation generally requires a simplifying framework (or an organizing principle) to be imposed either at the weak scale or the high scale. Even with the varied simplified frameworks that have been traditionally used, such as supergravity-motivated models, gauge-mediated models, and so on, there are a sufficient number of parameters that one usually resorts to numerical sampling. While this approach usually gives the correct general trends, it could also easily give misleading results if the physics of the model depends very sensitively on a sampled parameter.

Disentangling the dependencies of any given supersymmetric parameter on other parameters has proved to be formidable task. The first step, extracting weak scale observables, is likely going to be challenging both experimentally (discovery, and then mass and coupling measurements) and theoretically (mixings between gauginos, one-loop corrections, etc.). Hence, the subsequent extrapolation of the full theory to higher scales will be unreliable unless quantities can be found that are not highly interdependent on other parameters of the theory. One such quantity is the ratio of the gaugino mass to the gauge coupling squared, $M_{a} / \alpha_{a}$, where $a=1,2,3$ corresponds to $U(1)_{Y}, S U(2)_{L}, S U(3)_{c}$. In principle, one can extract $M_{a} / \alpha_{a}$ by "merely" measuring the gaugino mass, given that the standard model gauge couplings are by now well measured. Remarkably, the one-loop RG equation for $M_{a} / \alpha_{a}$ vanishes [13], which suggests that a measurement at the weak scale would determine the value at the high scale, up to small two-loop corrections (albeit scaled by $\left.\sim \ln Q_{\text {high }} / Q_{\text {weak }}\right)$.

The usefulness of the ratio $M_{a} / \alpha_{a}$ at higher scales is, ultimately, model-dependent. However, there are strong motivations to think that the pattern of the gauge couplings and the gaugino masses at the high scale will determine a great deal about the high scale theory. In GUTs, one ordinarily expects that the gauge couplings and the gaugino masses will "unify", or take the same value, after being embedded in the GUT group [14]. Even in string theory there are reasons to suggest the gaugino masses could be unified in the simplest scenarios [15]. If we could reliably calculate $M_{a} / \alpha_{a}$ at the high scale, no doubt one could match string phenomenology to such quantities, strengthening the predictions of successful models (and eliminating classes of unsuccessful models). In addition, given a motivation for thinking $g_{\mathrm{GUT}}$ or $g_{\text {string }}$ should take 
a certain value, it would also be trivial to extract the gaugino masses by themselves from the ratios $M_{a} / \alpha_{a}$.

The difficulty of unambiguously extrapolating the individual quantities $g_{a}$ and $M_{a}$, or even other weak scale parameters such as squark and slepton soft masses, is mainly due to the oneloop RG evolution and one-loop dependence on threshold corrections. In particular, extrapolating up 13 orders of magnitude in scale to the apparent unification scale $M_{\text {unif }} \approx 2 \times 10^{16} \mathrm{GeV}$ is notoriously complicated by weak scale supersymmetry threshold corrections, GUT scale threshold corrections, extra matter at intermediate scales, Planck scale corrections, etc. Using the ratio $M_{a} / \alpha_{a}$ mitigates many of these issues due to the one-loop RG invariance, as we will see.

Our goal is ultimately to determine how well $M_{a} / \alpha_{a}$ can be known at higher scales, and thus we will be primarily concerned with running up to the high scale (or messenger scale), rather than the more traditional approach of running down from a boundary condition imposed by some supersymmetry breaking ansatz. Our results can, of course, be easily inverted should there be a motivation to do so. Bottom-up approaches to supersymmetry have been considered before (see e.g. [17, 16, 17, 18]), generally with the philosophy that weak scale phenomenology ought to be extrapolated to the high scale, leaving the high scale matching to GUTs or string theory. Our approach is similar, in that we start from the weak scale, but differs from some of the previous analyses by extrapolating only the ratios $M_{a} / \alpha_{a}$, and yet also considering various high scale effects. Throughout most of this work we illustrate the results of extrapolation from near the weak scale $\sim 1 \mathrm{TeV}$ up to the apparent unification scale $\sim 10^{16} \mathrm{GeV}$; should the soft mass generation scale be much lower as in gauge-mediated supersymmetry breaking models, the size of the corrections will be smaller, but can still be easily extracted from most of the graphs we present. Similarly, effects in high scale theories (with gravity-mediated supersymmetry breaking) can give corrections to the ordinary expectations for $M_{a} / \alpha_{a}$ at the weak scale. By quantifying the various effects that could disrupt the one-loop expectations for $M_{a} / \alpha_{a}$, we are simultaneously showing that a naive interpretation of the gaugino mass ratios at the weak scale could easily give misleading conclusions, but a systematic evaluation of the effects discussed here could lead to intriguing signals of physics at much higher scales.

The most obvious class of corrections to the one-loop invariant $M_{a} / \alpha_{a}$ are the two-loop corrections [19, 20, 21] (in the supersymmetric $\overline{\mathrm{DR}}$ renormalization scheme [22]). The two-loop

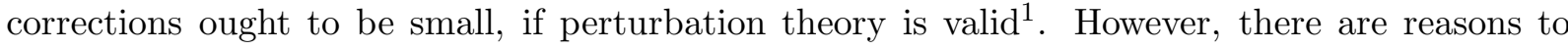
suggest that the size of two-loop corrections could be larger than one might naively guess. The two-loop coefficients are not of $\mathcal{O}(1)$, but in fact $\mathcal{O}(10)$ (in the minimal supersymmetric standard model (MSSM) the two-loop coefficients lie in the range $9 / 5 \leq B_{a b}^{(2)} \leq 25$ ). Also, two-loop terms proportional to the Yukawa couplings are present, and of course the top Yukawa is large at the weak scale. Finally, extrapolation to the high scale involves running over 13 orders of magnitude in scale, and therefore the (resummed) logarithm from RG evolution is comparatively large. (Analogously, if the soft mass generation scale is much lower, then the effect of RG evolution is not nearly as large.)

If two-loop corrections become important, then three-loop corrections may also be important, if for no other reason than to check that perturbation theory is indeed valid. However, a complete

\footnotetext{
${ }^{1}$ Which we assume throughout this paper.
} 


\begin{tabular}{r|ccc} 
& One-loop & Two-loop & Three-loop \\
\hline$g_{i}$ & $g_{i}$ & $g_{a}, Y_{x}$ & $g_{a}, Y_{x}$ \\
$Y_{w}$ & $Y_{x}, g_{a}$ & $Y_{x}, g_{a}$ & $Y_{x}, g_{a}$ \\
$M_{i}$ & $M_{i}, g_{i}$ & $M_{a}, g_{a}, Y_{x}, A_{x}$ & $M_{a}, g_{a}, Y_{x}, A_{x}$ \\
$A_{w}$ & $A_{x}, g_{a}, Y_{x}, M_{a}$ & $A_{x}, g_{a}, Y_{x}, M_{a}$ & $A_{x}, g_{a}, Y_{x}, M_{a}$
\end{tabular}

Table 1: Dynamical parameter dependence in supersymmetry for one-, two-, and three-loop renormalization group equations. In the MSSM the labels $i, a=1,2,3$ specify gauge couplings and $w, x=u, d, e$ specify Yukawa couplings, with $i$ and $w$ fixed while $a$ and $x$ are summed over.

three-loop analysis would entail using three-loop gauge and gaugino mass $\beta$-functions, at least two-loop Yukawa couplings and scalar trilinear couplings, and two-loop thresholds. The threeloop gauge coupling $\beta$-functions for a general MSSM were presented in Ref. [23], and the threeloop gaugino $\beta$-functions were recently presented in Ref. [24]. Two-loop $\beta$-functions for the Yukawa couplings and scalar trilinear couplings are by now well known [25, 26]. To the best of our knowledge two-loop threshold corrections (in a nondecoupling scheme) have not been calculated, although one could implement a decoupling scheme whereby the two-loop coefficients are changed as each threshold is crossed; this procedure is an approximation that should be straightforward to calculate from generalized two-loop $\beta$-functions, although we decline to present it here. An additional well-known complexity in going to three-loops is that the $\beta$-functions are no longer scheme independent [27]. In any case, we will not attempt to calculate corrections beyond twoloop, instead relying on others' calculations [23, 28] of three-loop corrections to guide us in those cases where we suspect perturbation theory may be in trouble.

The central reason why three-loop corrections should be unnecessary (unless a coupling gets large) is that there is no dependence on any additional parameters beyond those needed for the two-loop corrections. The intriguing pattern of the supersymmetric parameter interdependence is given in Table 1, where we show the dependencies arising at one-, two-, and three-loop order in the RG equations. The results to three-loop have been explicitly calculated for the gauge couplings $g$, Yukawa couplings $Y$, and gaugino masses $M$ [23, 24], and we suspect the three-loop result for the scalar trilinear couplings $A$ holds based on two-loop results 25, 26].

The parameter dependencies in Table 1 are central to our analysis. They imply, specifically, that the set of parameters $(g, M, Y, A)$ are sufficient to compute RG evolution to three-loop (and probably to all orders), and that we need not be concerned with the squark or slepton soft masses or the bilinear couplings (such as $\mu$ or $B$ ) directlyf?. This interdependence does, however, deserve a few further comments.

In the exact supersymmetric limit, only gauge couplings and superpotential parameters (including Yukawa couplings) remain. It is therefore not surprising that the gauge couplings and Yukawa couplings depend on each other, but not on any soft breaking parameter. That the soft breaking parameters $M$ and $A$ depend on only the four parameters $(g, M, Y, A)$ can ultimately

\footnotetext{
${ }^{2}$ The extraction of the scalar trilinear couplings at the weak scale, however, can presumably be obtained only by measuring the off-diagonal elements of the sfermion mass matrices.
} 
be related to theoretical properties of the superpotential. There has been a significant renewed interest in exact $\beta$-functions in $(N=1)$ supersymmetric theories. Using a fully Wilsonian treatment, exact one-loop $\beta$-functions for the gauge couplings [29] and gaugino masses [30, 24] can be found by expressing the soft breaking masses as spurions and then exploiting the holomorphy of the Lagrangian. Although these results are not directly useful for calculations with canonically normalized fields, they do allow the computation of higher order $\beta$-functions including some of the three-loop results mentioned above.

The outline of the paper is as follows. In Sec. 2, we present two-loop effects in the MSSM. We discuss the two-loop RG equations including both the "pure gaugino" and Yukawa terms, and the dependencies on the sign of the scalar trilinear coupling as well as the gaugino mass. We also discuss weak scale threshold corrections and superoblique corrections to the ratio $M_{a} / \alpha_{a}$. In Sec. 3 we discuss a series of effects beyond the MSSM, including an extra $U(1)^{\prime}$. Consistently adding an extra $U(1)^{\prime}$ to the gauge structure of the MSSM requires the nontrivial cancellation of $U(1)^{\prime}$ anomalies, and we relegate of discussion of this to Appendix B. We consider two models (with anomaly cancellation); a minimal $U(1)^{\prime}$ model and an $E_{6}$ model. We also consider adding extra matter to the theory, and its effect on the ratio $M_{a} / \alpha_{a}$. In Sec. 1 we discuss high scale corrections to the ratio $M_{a} / \alpha_{a}$ from GUT threshold corrections, effects due to breaking GUTs at other scales, Planck scale corrections, and supergravity effects. In Sec. 5 we discuss the consequences of extra matter with supersymmetry breaking masses that induce "corrections" via gauge-mediation. Finally, in Sec. 6 we present our conclusions. In Appendix A, we write the full two-loop RG equations for the gauge couplings and gaugino masses including all Yukawa couplings appropriate to the model, and then apply the results to the MSSM, MSSM $+U(1)^{\prime}$ model, and an $E_{6}$ model.

\section{Effects in the MSSM}

The first class of corrections to consider are those arising from the "standard" two-loop terms in the RG equation for $M_{a} / \alpha_{a}$. This will be done by starting with certain restrictive assumptions about the initial conditions and the two-loop terms to gain insight into the corrections, and then removing the assumptions one by one. Both weak scale threshold corrections and superoblique corrections are considered here, since they are generic in any weak scale supersymmetric model.

\subsection{Two-loop effects in the MSSM}

To begin and start with a point of reference, consider the RG evolution of $M_{a} / \alpha_{a}$ using the two-loop RG equations in the MSSM. The RG equation for the ratio $M_{a} / \alpha_{a}$ is given in Eq. (42), and throughout much of the following discussion we use "pure gaugino" and "Yukawa" contributions to refer to the first and second set of terms in the square brackets of the RG equation. First, take the unrealistic case where the Yukawa couplings are set to zero, which will allow us to analyze the pure gaugino contributions to the RG equations with the least number 


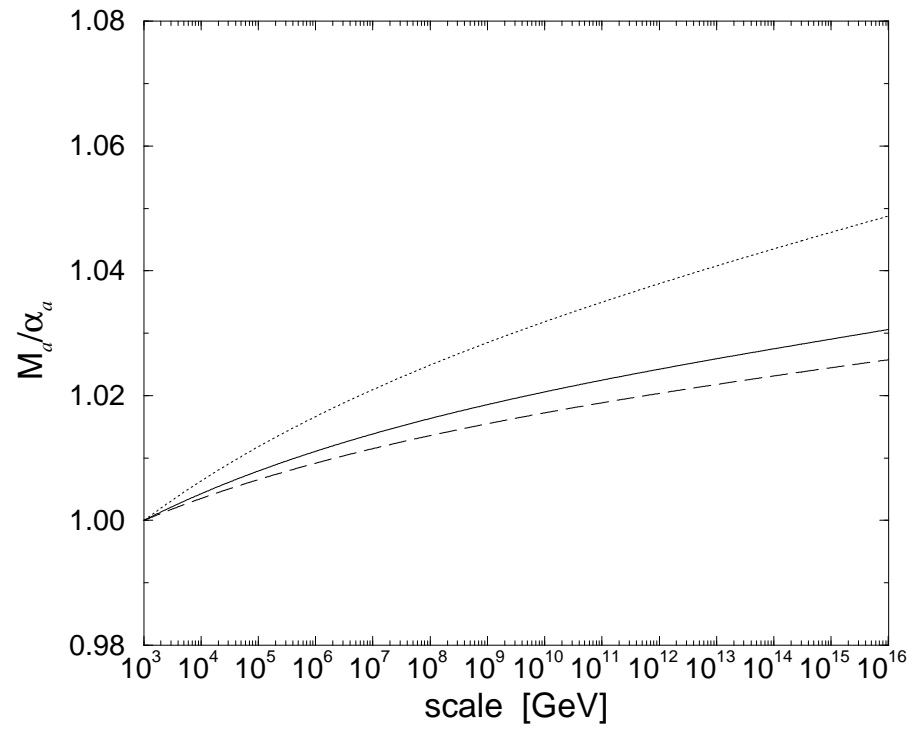

Figure 1: Evolution of $M_{a} / \alpha_{a}$ as a function of scale. The solid, dotted, and dashed lines correspond to $a=1,2,3$ respectively. Note that we have normalized the $y$-axis by dividing by the value $M_{1} / \alpha_{1}$ at $1 \mathrm{TeV}$.

of complexities. Furthermore, let's start by assuming that

$$
M_{1} / \alpha_{1}=M_{2} / \alpha_{2}=M_{3} / \alpha_{3}
$$

holds at a scale $Q$ near the weak scale, but above all weak scale thresholds (these will be discussed in Sec. 2.2). The evolution of the gauge couplings and gaugino masses is governed by six coupled RG equations (see Appendix A), but this is still analytically rather complicated. In Fig. 1, we show the evolution of the ratio $M_{a} / \alpha_{a}$ from $10^{3} \rightarrow 10^{16} \mathrm{GeV}$. By choosing to start the running at $1 \mathrm{TeV}$ with Eq. (1) as the weak scale boundary condition, we are restricting $M_{1}(1 \mathrm{TeV}) \lesssim 190$ $\mathrm{GeV}$, to prevent the gluino mass $M_{3}(1 \mathrm{TeV})$ from exceeding $1 \mathrm{TeV}$. For smaller values of $M_{1}$ (and correspondingly $M_{2}$ and $M_{3}$ ), the results are identical since there is only one scale in the problem (any one of the gaugino masses determines the scale). Nevertheless, for definiteness we take $M_{1}(1 \mathrm{TeV})=100 \mathrm{GeV}, M_{2}(1 \mathrm{TeV}) \approx 190 \mathrm{GeV}$, and $M_{3}(1 \mathrm{TeV}) \approx 527 \mathrm{GeV}$ in the following unless otherwise specified. The latter values $\left(M_{2}\right.$ and $\left.M_{3}\right)$ are considered mildly approximate since their determination requires the gauge couplings at $1 \mathrm{TeV}$, and therefore subject to weak scale threshold corrections.

There are a few important remarks to be made based on this simple exercise. First, the size of the pure gaugino corrections to the one-loop invariant $M_{a} / \alpha_{a}$ are less than $5 \%$ after scaling 13 orders of magnitude. In all cases the correction is a slight increase with increasing scale (RG equation is positive), reflecting the usual choice of phases in the initial condition, Eq. (1). The most striking feature is that the size of the two-loop pure gaugino corrections are, in order from largest to smallest (at any scale), $M_{2} / \alpha_{2}, M_{1} / \alpha_{1}, M_{3} / \alpha_{3}$. That such an ordering should be expected can be seen by examining the RG equation for the difference of two ratios (again, 
Yukawa couplings set to zero),

$$
\frac{d}{d t}\left(\frac{M_{a}}{g_{a}^{2}}-\frac{M_{b}}{g_{b}^{2}}\right)=\frac{2}{\left(16 \pi^{2}\right)^{2}} \sum_{c}\left(B_{a c}^{(2)}-B_{b c}^{(2)}\right) g_{c}^{2} M_{c}
$$

where the differences are

$$
\begin{aligned}
& B_{2 c}^{(2)}-B_{1 c}^{(2)}=\left(-\frac{154}{25}, \frac{98}{5}, \frac{32}{5}\right) \\
& B_{1 c}^{(2)}-B_{3 c}^{(2)}=\left(\frac{144}{25},-\frac{18}{5}, \frac{18}{5}\right)
\end{aligned}
$$

using the two-loop coefficients given in Appendix A. Since the difference between the two-loop coefficients is positive for the dominant $g_{3}^{2} M_{3}$ term, the $\mathrm{RG}$ equations for the difference of the ratios $\left(M_{2} / \alpha_{2}-M_{1} / \alpha_{1}\right)$ and $\left(M_{1} / \alpha_{1}-M_{3} / \alpha_{3}\right)$ are also positive, and thus $M_{2} / \alpha_{2}$ obtains the largest two-loop correction, followed by $M_{1} / \alpha_{1}$ and $M_{3} / \alpha_{3}$. This ordering holds if $g_{2}^{2} M_{2} \lesssim g_{3}^{2} M_{3}$, and it would only be if $g_{2}^{2} M_{2} \gg g_{3}^{2} M_{3}$ and/or $g_{1}^{2} M_{1} \gg g_{2}^{2} M_{2}, g_{3}^{2} M_{3}$ that one would expect the ordering of the size of the two-loop corrections to be different.

The next stage is to restore the two-loop terms proportional to the Yukawa couplings. In the RG equation for the ratio $M_{a} / \alpha_{a}$, each Yukawa coupling (squared) is multiplied by the associated soft breaking term, the scalar trilinear coupling, which provides the mass scale. Unlike the "pure gaugino" terms, additional scales independent of the gaugino masses can partially determine the RG evolution. Potentially, one new scale is introduced for every nonzero Yukawa coupling. However, we will assume that the $3 \times 3$ Yukawa coupling matrices $\mathbf{Y}_{u}, \mathbf{Y}_{d}$, and $\mathbf{Y}_{e}$ (in flavor space) can be reduced to the dominant third generation couplings $Y_{t}, Y_{b}$, and $Y_{\tau}$, and consequently the only relevant scalar trilinear couplings are $A_{t}, A_{b}$, and $A_{\tau}$. The RG equations for the Yukawa couplings and scalar trilinear couplings are given in Appendix A.3. The size of the terms proportional to the Yukawa couplings depend on the competition between terms proportional $g^{2} M$ versus those proportional to $Y^{2} A$. It is therefore important to recognize that the two-loop corrections to $M_{a} / \alpha_{a}$ need not be proportional to (or suppressed by) a gauge coupling squared, but instead a Yukawa coupling squared.

The Yukawa couplings are extracted at the weak scale from the fermion mass $m(m)$, but they also depend on $\tan \beta$, the ratio of vacuum expectation values of the two neutral Higgs doublet scalar fields. The perturbative lower and upper limits on $\tan \beta$ can be found by successfully extrapolating the Yukawa couplings up to the high scale $Q_{\text {high }}$ without encountering a Landau pole. We will be considering three cases in the following: one case each of low and high $\tan \beta$, and in subsequent discussion we usually take an intermediate $\tan \beta$ value.

In the low and intermediate $\tan \beta$ cases, we can safely neglect the terms proportional to $Y_{b}$ and $Y_{\tau}$. If we once again use the initial condition Eq. (四), normalizing to $M_{3} / \alpha_{3}$ at $Q=1 \mathrm{TeV}$, then there are only two additional parameters, $A_{t}$ and $\tan \beta$. We could just as easily substitute $\tan \beta$ for $Y_{t}\left(Q_{\text {high }}\right)$, which is probably a better calculational input parameter since it is not sensitively dependent on initial conditions, thresholds, and the loop order of the Yukawa RG equations, etc. Of course to precisely translate a $Y_{t}\left(Q_{\text {high }}\right)$ back into a $\tan \beta$, one must treat the above carefully. We will provide approximate $\tan \beta$ values corresponding to particular choices of $Y_{t}\left(Q_{\text {high }}\right)$. 


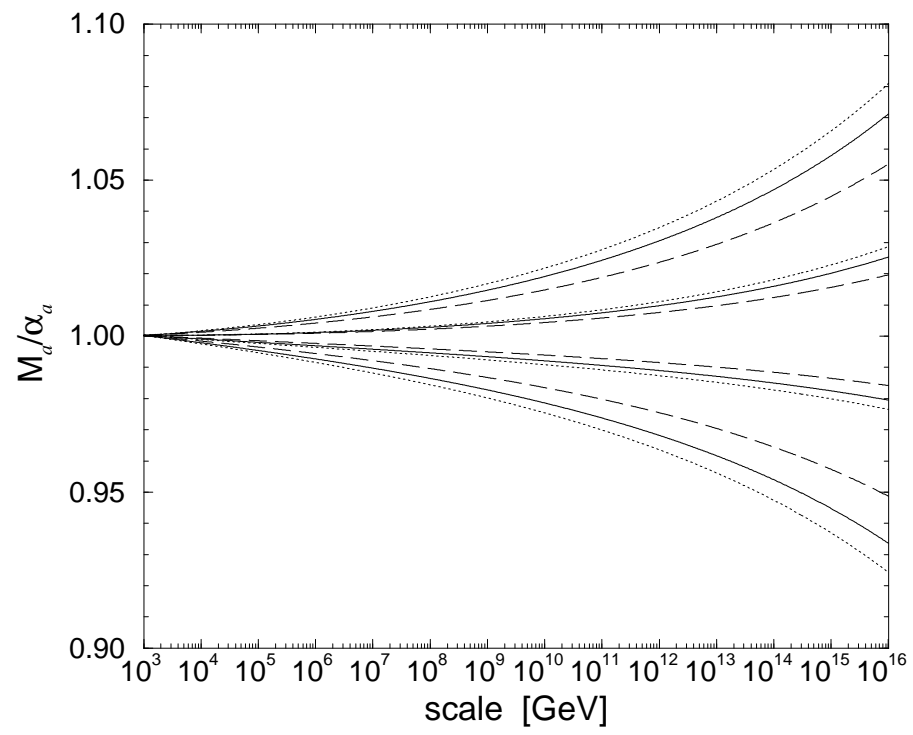

Figure 2: Evolution of $M_{a} / \alpha_{a}$ as a function of scale, for $Y_{t}\left(10^{16} \mathrm{GeV}\right)=1$, and $A_{t}=$ $M_{3}, 0,-M_{3},-2 M_{3}$ at $Q=1 \mathrm{TeV}$ for each triplet of (solid, dotted, dashed) lines from top to bottom. As in Fig. 1, the solid, dotted, and dashed lines correspond to $a=1,2,3$ respectively. The normalization differs from Fig. 1 in that the ratio $M_{a} / \alpha_{a}$ with Yukawa couplings included is normalized against the ratio without Yukawa couplings (identical to the curves in Fig. 1).

Figure 2 shows the effect of a nonzero Yukawa coupling (and scalar trilinear coupling), with $Y_{t}\left(10^{16} \mathrm{GeV}\right)=1$, corresponding to $\tan \beta \approx 2.1$ using one-loop evolution of the top Yukawa coupling. [Two-loop corrections to the top Yukawa coupling typically alter the value of $\tan \beta$ by about -0.05 to maintain $Y_{t}\left(10^{16} \mathrm{GeV}\right)=1$.] The results for $M_{a} / \alpha_{a}$ were normalized by dividing by the ratio $M_{a} / \alpha_{a}$ extracted from an equivalent model with the Yukawa couplings set to zero (i.e., the results shown in Fig. [1). The effects of the Yukawa coupling terms are therefore evident separately from the pure gaugino terms that enter at two-loop. Since it is possible for the gaugino masses and gauge couplings to be established experimentally without any detailed knowledge of the scalar trilinear couplings, the two-loop corrections in Fig. 1 are calculable and do not pose a fundamental theoretical uncertainty in extrapolating to the high scale (unlike the scalar trilinear couplings). This provides additional motivation to normalize against the curves in Fig. 1.

It is clear from Fig. 2 that different choices for $A_{t}(1 \mathrm{TeV})$ affect the two-loop running at the level of nearly $10 \%$, if a Yukawa coupling is $\mathcal{O}(1)$. In the cases where $A_{t}>0$ and $A_{t}<-2 M_{3}$, the scalar trilinear coupling runs to values at the high scale that are more than a factor of 10 larger than $M_{3}(1 \mathrm{TeV})$. These input parameters may pose serious problems related to finetuning (squark masses will be driven to similarly large values), and may also be untenable if a charge or color breaking vacuum is encountered at the weak scale. To see how large the Yukawa coupling and trilinear scalar coupling need to be to get a significant correction, consider simply the competition between the top Yukawa term $C_{a}^{u} Y_{t}^{2} A_{t}$, and the gluino mass term $B_{a 3}^{(2)} g_{3}^{2} M_{3}$. One immediately observes that the Yukawa terms suffer from suppression in the overall constants: $B_{a 3}^{(2)} / C_{a}^{u}=\frac{44}{13}, 4, \frac{7}{2}$ for $a=1,2,3$ in the MSSM. If $g_{3}$ and $Y_{t}$ are of the same order, then $A_{t} \sim 4 M_{3}$ 


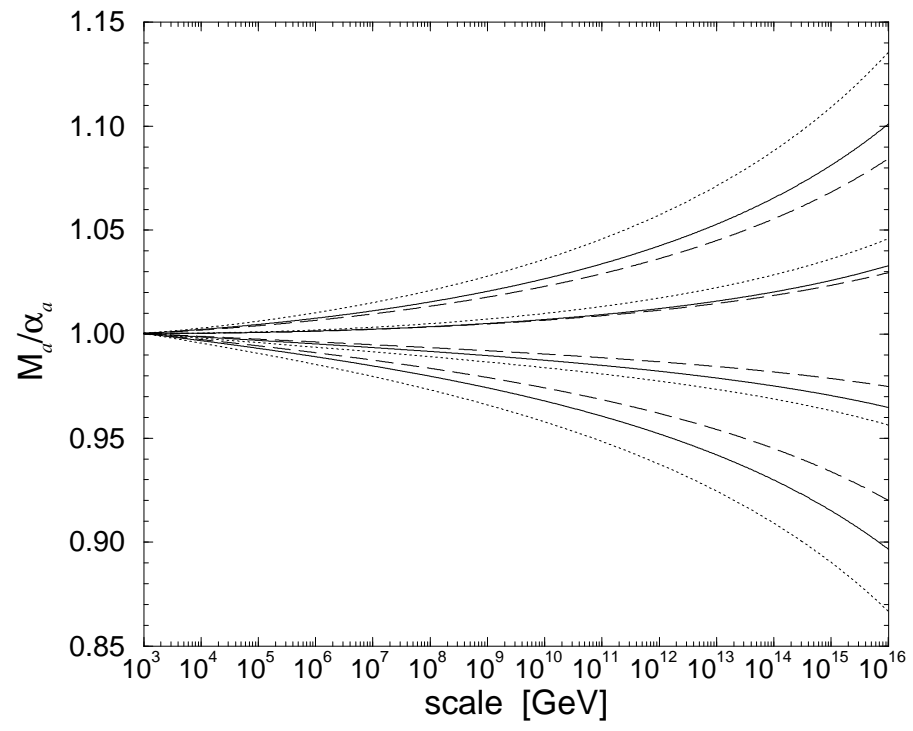

Figure 3: Analogous to Fig. 2, with $Y_{\tau}\left(10^{16} \mathrm{GeV}\right) \approx Y_{b}\left(10^{16} \mathrm{GeV}\right)=1$, and the choices $A_{t}=$ $A_{b}=A_{\tau}=M_{3}, 0,-M_{3},-2 M_{3}$ for each triplet of lines from top to bottom.

to instigate corrections of the same order as those of the pure gaugino terms.

In the case with large $\tan \beta$, both $Y_{b}$ and $Y_{\tau}$ evolve to large values, and thus all Yukawa coupling terms must be included. In Fig. 3 we show the ratios $M_{a} / \alpha_{a}$ normalized as in Fig. 2, with $Y_{\tau}\left(10^{16} \mathrm{GeV}\right) \approx Y_{b}\left(10^{16} \mathrm{GeV}\right)=1$ corresponding to $\tan \beta \approx 55$, and the choices $A_{t}=A_{b}=$ $A_{\tau}=M_{3}, 0,-M_{3},-2 M_{3}$ at $1 \mathrm{TeV}$. The size of the correction is somewhat larger than in Fig. 2, up to nearly $15 \%$, mainly because all three Yukawa terms are constructively contributing to the $\beta$-function.

Up to now we have been considering the evolution assuming the initial condition Eq. (11). Although the simplest supergravity models and gauge-mediated models suggest such a relation should hold near the weak scale, it is nevertheless prudent to study other alternatives (four quite different examples of models with that are not expected to have "unified" gaugino masses can be found in Refs. [31, 32, 28, 33]). Consider a model with gaugino masses $M_{a}^{\prime} \equiv k_{a} M_{a}$, such that

$$
\frac{k_{1} M_{1}}{\alpha_{1}} \neq \frac{k_{2} M_{2}}{\alpha_{2}} \neq \frac{k_{3} M_{3}}{\alpha_{3}}
$$

while Eq. (1) holds for $M_{a}$. In essence, we are considering two distinct models with different boundary conditions on the gaugino masses at the weak scale. The scaling factors $k_{a}$ relate a model that does respect the initial condition, Eq. (11), to one that does not. If $M_{a}^{\prime}=k_{a} M_{a}$ is to hold for all scales, then the scaling factors must depend on the renormalization scale. The renormalization group equation for the scaling factors can be obtained from the RG equations for the gaugino masses and gauge couplings,

$$
\frac{d}{d t} k_{a}=\frac{2 g_{a}^{2}}{\left(16 \pi^{2}\right)^{2}}\left[\sum_{b} B_{a b}^{(2)} g_{b}^{2} \frac{M_{b}}{M_{a}}\left(k_{b}-k_{a}\right)+\sum_{x} C_{a}^{x} \frac{A_{x}}{M_{a}}\left(\frac{A_{x}^{\prime}}{A_{x}}-k_{a}\right) \operatorname{tr}\left(Y_{x}^{\dagger} Y_{x}\right)\right]
$$


which represents the evolution of the fraction

$$
\frac{M_{a}^{\prime}}{M_{a}}=\frac{M_{a}^{\prime} / \alpha_{a}}{M_{a} / \alpha_{a}}
$$

If we take only one $k_{a}$ to be not equal to one, and approximate $A_{x}^{\prime} / A_{x} \sim 1$, Eq. (四) becomes

$$
\frac{d}{d t} k_{a}=\left(1-k_{a}\right) \frac{2 g_{a}^{2}}{\left(16 \pi^{2}\right)^{2}}\left[\sum_{b ; b \neq a} B_{a b}^{(2)} g_{b}^{2} \frac{M_{b}}{M_{a}}+\sum_{x} C_{a}^{x} \frac{A_{x}}{M_{a}} \operatorname{tr}\left(Y_{x}^{\dagger} Y_{x}\right)\right] .
$$

Technically, this is only an approximate RG equation that works well for $k_{1}$ or $k_{2}$, and to a lesser extent $k_{3}$. This is because RG equations for the scalar trilinear couplings depend on the gaugino masses, and we have assumed the scalar trilinear couplings are identical for both models at all scales to construct Eq. (6). However, typically the correction is not very large, and thus Eq. (6) is a reasonable approximation to the "true" ratio $\left(M_{a}^{\prime} / \alpha_{a}\right) /\left(M_{a} / \alpha_{a}\right)$.

By comparing the two models using the scaling factors $k_{a}$, some analytical insight can be gained into the effect of the nonstandard initial condition in Eq. (3) on the resulting high scale values. The RG equation, Eq. (6), indicates that $k_{a}$ will tend toward 1 with increasing scale if $A>0$ or if simply the pure gaugino terms (positively) dominate over the Yukawa terms. To gain further insight into Eq. (6), we can approximately solve for $k_{a}$ by substituting "average" values for the quantities dependent on the renormalization scale, giving the constant $Z_{k}$ which we schematically write as

$$
Z_{k}=\bar{g}_{a}^{2}\left[\sum_{b ; b \neq a} B_{a b}^{(2)} \bar{g}_{b}^{2} \frac{\bar{M}_{b}}{\bar{M}_{a}}+\sum_{x} C_{a}^{x} \frac{\bar{A}_{x}}{\bar{M}_{a}} \operatorname{tr}\left(\bar{Y}_{x}^{\dagger} \bar{Y}_{x}\right)\right]
$$

Then the solution to Eq. (6) becomes

$$
k_{a}\left(Q_{f}\right)=1+\left(k_{a}\left(Q_{i}\right)-1\right)\left(\frac{Q_{i}}{Q_{f}}\right)^{\frac{Z_{k}}{128 \pi^{4}}} .
$$

With sufficiently small $Q_{i} / Q_{f}$ and $Z_{k}>0$, it is obvious that $k_{a}$ converges to one as the scale is increased. Alternatively, with sufficiently small $Q_{i} / Q_{f}$ and $Z_{k}<0, k_{a}$ will diverge either toward very small or negative values (if initially $k_{a}<1$ ) or very large values (if initially $k_{a}>1$ ). The mundane, but more likely possibility is that

$$
\left(\frac{Q_{i}}{Q_{f}}\right)^{\frac{Z_{k}}{128 \pi^{4}}} \sim 1
$$

and so the initial conditions in Eq. (3) are preserved under RG evolution. For $Q_{i} / Q_{f}=10^{-13}$, we find that $Z_{k}$ must be

$$
Z_{k} \gtrsim(20,100) \quad \text { for } \quad\left(\frac{Q_{i}}{Q_{f}}\right)^{\frac{Z_{k}}{128 \pi^{4}}} \lesssim(0.95,0.8),
$$




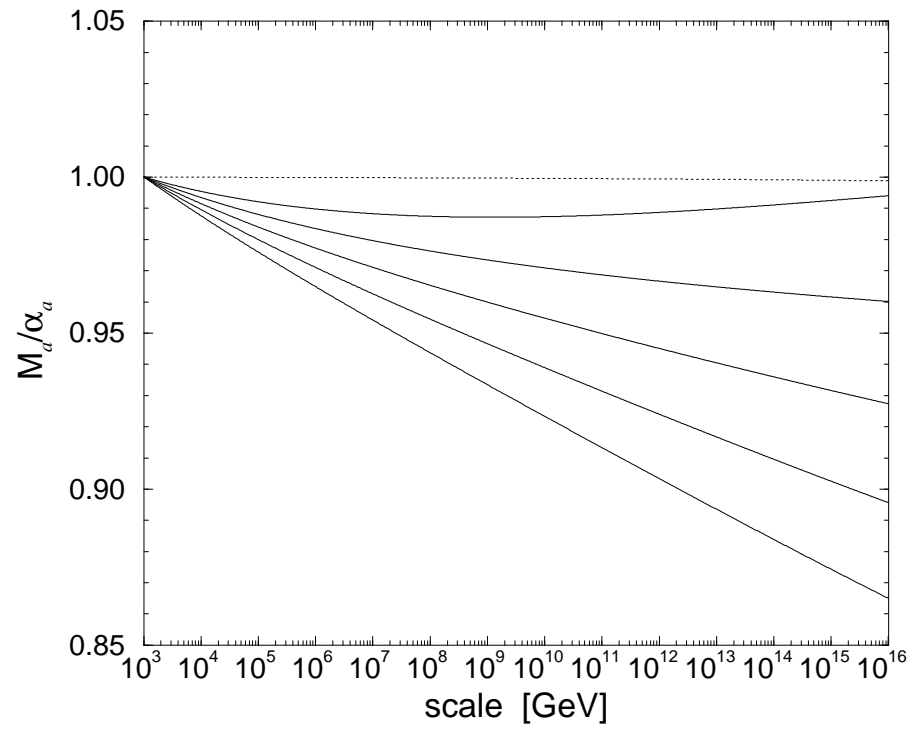

Figure 4: The ratio $M_{a} / \alpha_{a}$ is plotted versus scale, for a model with $\tan \beta=5$ and $\left(-M_{1} / \alpha_{1}\right)=$ $M_{2} / \alpha_{2}=M_{3} / \alpha_{3}$ at $1 \mathrm{TeV}$. The curves are normalized by dividing by a model with the identical initial conditions, except the sign of $M_{1}$ is positive. The set of solid lines correspond to the normalized $\left(-M_{1} / \alpha_{1}\right)$, with $A_{x}(1 \mathrm{TeV})=2\left|M_{3}\right|,\left|M_{3}\right|, 0,-\left|M_{3}\right|,-2\left|M_{3}\right|$ from bottom to top. The dotted line corresponds to the normalized ratios $M_{2} / \alpha_{2}$ and $M_{3} / \alpha_{3}$, which are independent of $A_{x}$.

and thus significant deviations to the initial conditions are not expected after running from the weak scale to the high scale. We have also confirmed this using numerical calculations. However, if $Z_{k}$ were very large and positive (a numerical value of several hundred), then $k_{a}$ will rapidly evolve toward 1 . To obtain a very large $Z_{k}$, one is faced with large two-loop corrections to the RG equations. To be sure perturbation theory is still valid, one should go to higher orders and check that the three-loop terms are indeed smaller than the two-loop terms. In Ref. [28] this was done for models with semi-perturbative unification, finding the interesting result that the initial condition on the gaugino mass ratios is not maintained under RG evolution, consistent with the above discussion.

Another possibility that can be examined with the formalism above is to allow $M_{1}$ and/or $M_{3}$ to be negative. This is possible because in general there can be nonzero phases associated with $M_{1}$ and $M_{3}$ (the phase of $M_{2}$ can be chosen to vanish). We limit ourselves to gaugino masses that are real, so that we can concentrate on merely a few different sign possibilities. To examine the impact of a particular sign choice on the RG evolution, assume the initial condition

$$
\pm \frac{M_{1}}{\alpha_{1}}=\frac{M_{2}}{\alpha_{2}}= \pm \frac{M_{3}}{\alpha_{3}}
$$

where we will examine the two cases $M_{1}<0, M_{2}, M_{3}>0$ and $M_{3}<0, M_{1}, M_{2}>0$. In Fig. 田 we plot the ratio of $M_{a} / \alpha_{a}$ in a model with $M_{1}<0$ normalized to an equivalent model with $M_{1}>0$, and all other initial conditions the same (we take the intermediate value $\tan \beta=5$ for the 


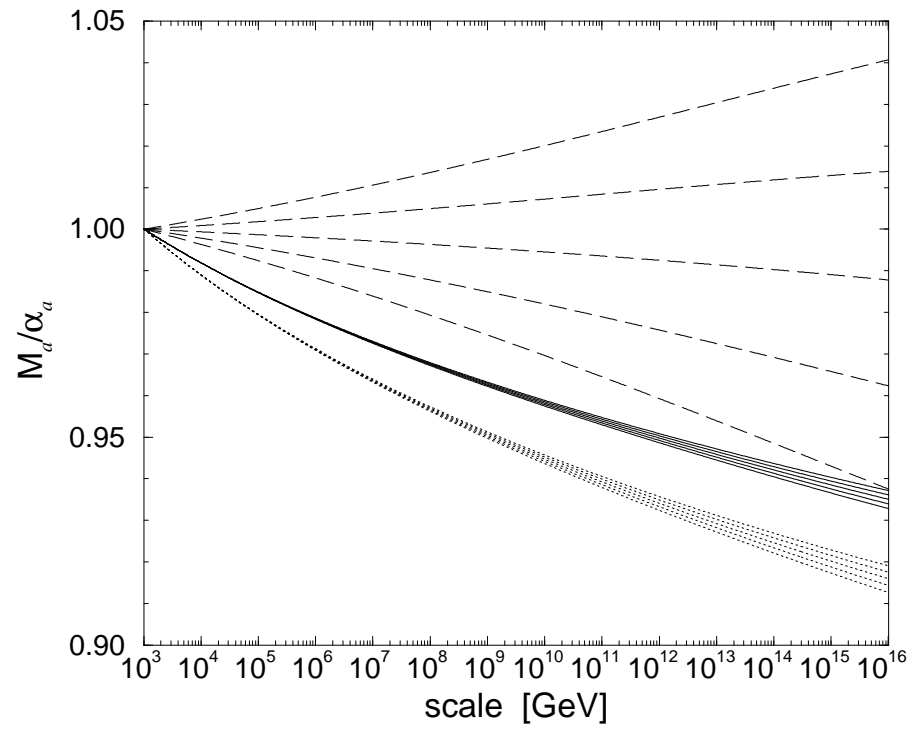

Figure 5: Analogous to Fig. 4, with $M_{1} / \alpha_{1}=M_{2} / \alpha_{2}=\left(-M_{3} / \alpha_{3}\right)$ at $1 \mathrm{TeV}$. The set of solid, dotted, and dashed lines correspond to the normalized $M_{1} / \alpha_{1}, M_{2} / \alpha_{2},\left(-M_{3} / \alpha_{3}\right)$, with $A_{x}(1 \mathrm{TeV})=2\left|M_{3}\right|,\left|M_{3}\right|, 0,-\left|M_{3}\right|,-2\left|M_{3}\right|$ from top to bottom for $\left(-M_{3} / \alpha_{3}\right)$, and from bottom to top for the other ratios.

purposes of this example). Since the dominant term in the evolution is $g_{3}^{2} M_{3}$, it is not surprising that $M_{2} / \alpha_{2}$ and $M_{3} / \alpha_{3}$ are virtually unaffected by the sign choice of $M_{1}$. However, $M_{1} / \alpha_{1}$ is significantly affected. Setting $k_{1}(1 \mathrm{TeV})=-1$, one finds that Eq. (6) captures essentially all of the difference between a model with and without a negative $M_{1}$. The size of the effect after running 13 orders of magnitude in scale varies depending on the competition between the pure gauge terms and the Yukawa terms in the RG equation. In the case where $A_{x}<0$, for example, there is a partial cancellation between the pure gaugino terms (dominated by $g_{3}^{2} M_{3}>0$ ) and the Yukawa terms, thus the RG evolution is not as pronounced.

In the case where $M_{3}<0$ and $M_{1}, M_{2}>0$, and the initial condition Eq. (11) holds, there is a more dramatic effect on the RG evolution of all the gaugino masses due to the dominance of the $g_{3}^{2} M_{3}$ term. In Fig. 奋 we illustrate the RG evolution for the same initial conditions as in Fig. 4, but with $M_{3}<0$ instead of $M_{1}<0$. We observe that the evolution of $\left(-M_{3} / \alpha_{3}\right)$ is not as significant as the evolution of $\left(-M_{1} / \alpha_{1}\right)$ (for this example), mainly because the $g_{3}^{2} M_{3}$ term is absent in Eq. (6) for $a=3$. (An equivalent way of thinking about this is that the $g_{3}^{2} M_{3}$ dominates the RG evolution for $M_{3} / \alpha_{3}$, which implies an approximate $M_{3}$ sign independence, see Eq. (42).) This also implies the Yukawa terms dominate over the pure gaugino terms in Eq. (6), giving the logarithmically increasing but well-defined separation between the different $\left(-M_{3} / \alpha_{3}\right)$ curves as $A_{x}$ is increased. However, we should point out that Eq. (6) only approximately accounts for the $\mathrm{RG}$ evolution of the ratio; this is because, as noted above, Eq. (16) does not account for the $M_{a}$ sign dependent part of the evolution of $A_{x}$. 


\subsection{Weak scale thresholds}

We have up to now avoided the issue of weak scale threshold corrections by judiciously choosing the initial conditions, including the gaugino masses, scalar trilinear couplings, etc., to hold at a scale above all weak scale thresholds. The motivation for this is that one can always match a weak scale theory including full threshold corrections to the initial conditions we gave in the examples above. In practice, this matching can become rather complicated [34, 35, 36] if one demands a high level of precision. However, it is important to understand the origin of the uncertainties associated with weak scale thresholds, as well as recognizing that, for example, measured (pole) masses must be translated into renormalized masses, and the corrections can be large (especially for the gluino [20, 34, 36]). Note that we have implicitly assumed the running gaugino masses are specified in the scheme appropriate for supersymmetry, namely dimensional reduction with modified minimal subtraction $(\overline{\mathrm{DR}})$ [22].

Threshold corrections arise from decoupling heavy particles from the spectrum by creating an effective theory without the heavy degrees of freedom that matches near the scale of the heavy particles. In weak scale supersymmetric theories one is interested in decoupling sparticles that are heavier than $M_{Z}$, the customary choice of scale for starting RG evolution since the gauge couplings are very well measured by LEP experiments. There are two methods for handling threshold corrections: (1) "decoupling method", where heavy particles are decoupled by altering the $\beta$-functions at the mass scale of the particles, and (2) "nondecoupling method", where fully supersymmetric $\beta$-functions are retained and the effects of heavy particles are "resummed" as corrections in the conversion from the measured to running quantity. Strictly speaking, the second method is best since full logarithmic and finite corrections with arbitrary mixing can be incorporated (although there are sometimes ways of re-incorporating finite corrections using the decoupling method). However, explicitly resumming logarithms using a nondecoupling method is really only useful when the scale of the decoupled particles is near the scale of the conversion from measured to running quantity, otherwise the first method should be used. For completeness, we give formulae to compute the one-loop $\beta$-function decoupling for the weak scale threshold corrections in Appendix A.2.

It is useful to first recall that the RG equation for the ratio $M_{a} / \alpha_{a}$ is independent of oneloop threshold corrections implemented as changes in the one-loop $\beta$-function coefficients. This is obvious from the RG equation, Eq. (42), since it is independent of $B_{a}^{(1)}$. Threshold corrections at two-loop would, however, change the coefficients for the ratio's RG equation. But, weak scale threshold corrections at two-loop are negligible when we are working with two-loop RG evolution equations [37], since no large logarithm develops (precisely because we demand the scale of the superpartner masses be near the weak scale). There are residual indirect effects from one-loop thresholds that arise because of the two-loop terms proportional to the gauge couplings, but in virtually all instances such corrections can be neglected.

Take the simplest case, the gluino mass [20, 34, 36]. It is easy to show that in the limit $m_{q} \ll m_{\tilde{g}} \ll m_{\tilde{q}}$ the logarithmic corrections scale with $\log m_{\tilde{q}}^{2} / m_{\tilde{g}}^{2}$ when the gluino (running) mass is evaluated at a scale $Q=m_{\tilde{g}}$. The coefficients of the log terms precisely match the shifts in the one-loop $\beta$-functions (see Appendix A.2). However, nonzero quark masses bring 
additional corrections, which can only be partially taken into account using a decoupling method. Furthermore, the corrections to the weak gaugino masses are complicated due to the mixings inherent in the resultant charginos and neutralinos [34, 36]. Luckily, incorporating the logarithmic corrections to the gluino mass, and incorporating approximate logarithmic corrections (neglecting mixings) in the weak gaugino masses is usually sufficient for most purposes. Since we specified running masses throughout the previous discussions, it was not necessary to explicitly calculate the corrections from translating the pole mass into the running $\overline{\mathrm{DR}}$ mass. However, experiments will ultimately measure the pole mass, and so these finite corrections must be taken into account. In general, extracting parameters from experiment will require a careful analysis by incorporating both finite corrections and logarithmic corrections (probably using a nondecoupling method as in Ref. 36]).

\subsection{Superoblique corrections}

There is further class of weak scale corrections [38, 39, 40, 41, 42, 43, 44] that result when supermultiplets are widely split in mass. These so-called "superoblique" corrections have much in common with oblique corrections of the standard model [41, 42], particularly because they do not decouple for scales smaller than the heaviest sparticle mass. The manifestation of superoblique corrections is the violation the relation $g=\tilde{g}$, where $g$ is the coupling of gauge bosons to fermions and scalars, and $\tilde{g}$ is the coupling of gauginos to a fermion and its scalar partner. Ordinarily supersymmetry enforces this relation to all orders (in a dimensional reduction scheme [20]), but since supersymmetry must be broken near the weak scale, differing corrections to $g$ and $\tilde{g}$ are expected.

Differentiating between the gauge and gaugino coupling suddenly begs the question of which one ought to be used in the ratio $M_{a} / \alpha_{a}$. To address this, there are four cases to be distinguished. To simplify the discussion, consider the effects of a single supermultiplet consisting of a scalar $\tilde{q}$ and its fermion partner $q$ with masses $m_{\tilde{q}}$ and $m_{q}$ respectively. (The extension to multiple multiplets and specialization to a particular gaugino is trivial.) The four cases are (1) $m_{\tilde{q}}, m_{q} \ll$ $M,(2) M \ll m_{\tilde{q}}, m_{q},(3) m_{q} \ll M \ll m_{\tilde{q}}$, and (4) $m_{\tilde{q}} \ll M \ll m_{q}$, where $M$ is the gaugino mass. The first case is trivial; it is easy to show that there is no distinction between $g$ and $\tilde{g}$ when all matter multiplets have masses well below that of the gaugino mass. The second case is nontrivial, and has been studied particularly for the messenger sector of gauge-mediated models [41, 44]. Even in cases when the lightest messenger scalar is 1/10 the mass of the messenger fermion, one finds the fractional difference $(g-\tilde{g}) / g \lesssim 10^{-4}$. It is really only the case where either a fermion is very heavy and scalars are light, or scalars are heavy and the fermion is light, when superoblique corrections are significant. Motivated by the current experimental bounds on the masses of the scalar partners that require $m_{q} \ll m_{\tilde{q}}$ for the MSSM matter multiplets? we will focus on the latter possibility.

The one-loop corrections to the self-energy of the gaugino are shown in Fig. 6. We are interested in the sfermion-fermion one-loop corrections that contain the superoblique corrections.

\footnotetext{
${ }^{3}$ With the possible exception of the scalar partner to the top quark.
} 


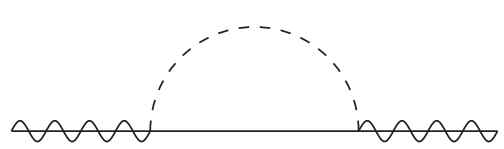

(a)

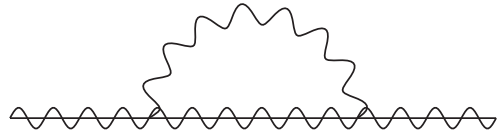

(b)

Figure 6: One-loop self-energy corrections to the gaugino mass from (a) sfermion-fermion contributions, and (b) gauge boson contributions.

The self-energy due to the sfermion-fermion diagram in Fig. 6(a) is

$$
\Sigma(\not p)=\frac{2 g^{2} S(q)}{16 \pi^{2}} \not p \int_{0}^{1} d x(1-x) \ln \frac{x m_{q}^{2}+(1-x) m_{\tilde{q}}^{2}-x(1-x) p^{2}}{\mu^{2}},
$$

where $p$ in the momentum of the gaugino, $\mu$ is the renormalization scale, and we have performed $\overline{\mathrm{DR}}$ subtraction of the infinite piece. The Dynkin index $S(q)$ is defined by $S(q) \delta^{a b} \equiv \operatorname{tr}_{q}\left(t^{a} t^{b}\right)$ in a normalization where $S(q)=\frac{1}{2}$ for the fundamental of $S U(N)$, and $S(q)=\frac{3}{5}(Y / 2)^{2}$ for $U(1)_{Y}$ in the GUT normalization. The correction to the running gaugino mass due to the superoblique corrections can be written as

$$
M(M)=M(M)_{0}\left(1+\frac{\alpha}{4 \pi} \sum_{q} A_{q}\right) .
$$

where $M(M)$ and $M(M)_{0}$ are the running $\overline{\mathrm{DR}}$ gaugino masses with (without) one-loop superoblique corrections applied. The function $A_{q}$ is

$$
A_{q}=2 S(q) \int_{0}^{1} d x(1-x) \ln \frac{x m_{q}^{2}+(1-x) m_{\tilde{q}}^{2}-x(1-x)\left[M(M)_{0}\right]^{2}}{\mu^{2}},
$$

approximating $\not p=M^{\text {pole }} \approx M(M)_{0}$. The above expression agrees with the squark-quark oneloop corrections calculated in Refs. [20, 44]. Note that there are additional one-loop finite corrections due to gauge boson loops [shown in Fig. 6(b)] that we do not present, since they can be absorbed into the translation between $M^{\text {pole }}$ and $M(M)$ [19, 20]. The correction to the gaugino coupling arises from wave-function renormalization $Z_{2}$ of the gaugino propagator $i Z_{2} /(\not p-M+i \epsilon)$, where $Z_{2}^{-1}=1-\frac{d}{d \not p} \Sigma(\not p)$ after expanding the denominator of the one-loop propagator $i /(\not p-M-\Sigma(\not p)+i \epsilon)$, in $(\not p-M)$. The result is a correction to the gaugino coupling

$$
\tilde{g}^{2}(M)=\tilde{g}_{0}^{2}(M)\left(1+\frac{\alpha}{4 \pi} \sum_{q} A_{q}\right) .
$$

using notation analogous to that in Eq. (13). (There are additional nonlogarithmic corrections [44] to Eq. (15), but they decouple when $M(M) \ll m_{\tilde{q}}$.) Thus, the ratio $M_{a} / \tilde{g}_{a}^{2}$ is independent of superoblique threshold corrections. This is an important result, because it means that using the coupling $g$ (that is more easily extracted from gauge boson interactions) to evolve the ratio $M_{a} / \alpha_{a}$ will reintroduce one-loop corrections for scales between $M<Q<m_{\tilde{q}}$. Alternatively, the uncertainty in translating $g$ into $\tilde{g}$ due to superoblique corrections is a fundamental uncertainty in evaluating the true one-loop invariant $M / \tilde{g}^{2}$ near the weak scale. 


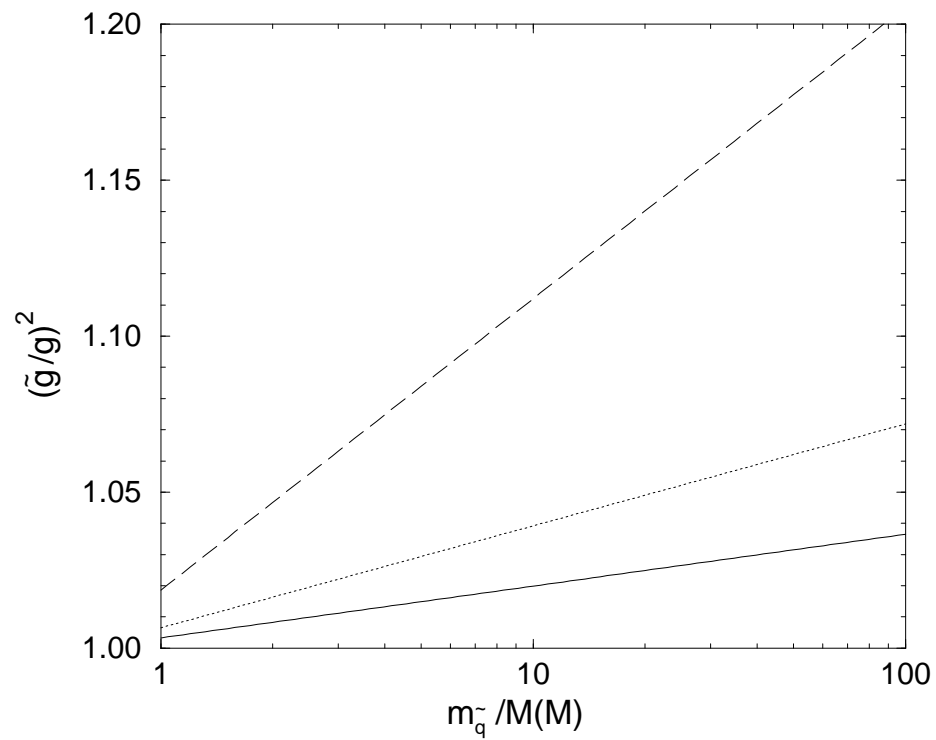

Figure 7: An example of superoblique corrections to $M_{a} / g_{a}^{2}$, due to setting the masses of the first two generations of squarks and sleptons to be $m_{\tilde{q}}$. The ratio $\left(\tilde{g}_{a} / g_{a}\right)^{2}=\left(M_{a} / g_{a}^{2}\right) /\left(M_{a} / \tilde{g}_{a}^{2}\right)$, where the denominator $M_{a} / \tilde{g}_{a}^{2}$ is independent of superoblique corrections, but the numerator $M_{a} / g_{a}^{2}$ is expected to be extracted from experiment. The solid, dotted, and dashed lines correspond to $a=1,2,3$. We assumed the initial condition, Eq. (11), with $M_{1}\left(M_{1}\right)=100 \mathrm{GeV}$, and evaluated the correction at the scale $M_{a}\left(M_{a}\right)$.

The size of the correction (including finite corrections) was given in Ref. [44, and takes the form

$$
\left(\frac{\tilde{g}}{g}\right)^{2}=1+\sum_{q} S(q) \frac{\alpha}{6 \pi}\left(\ln \frac{m_{\tilde{q}}^{2}}{Q^{2}}+\frac{11}{12}\right)
$$

where the sum is over all scalars $\tilde{q}$ transforming under the gauge group associated with $g$ (or $\tilde{g})$ with Dynkin index $S(q)$. Again, we are considering the case where the scalar partners are much heavier than the fermions. Of course we could have also considered the opposite case (see Refs. [11, 44]), but there is motivation, for example, to set some or all of the scalar partners of the first two generations in the MSSM to be very heavy, of order $\sim 20 \mathrm{TeV}$ or so [45, 46, 47]. These models avoid flavor changing neutral current (FCNC) constraints from experiment (that dominantly restrict the first two generations) by pushing the mass scale of the exchanged scalars sufficiently high so that universality and alignment are unnecessary. In these " $2-1$ " models the correction becomes 41, 44,

$$
\left(\frac{\tilde{g}_{a}}{g_{a}}\right)^{2}=1+\frac{2 \alpha_{a}}{3 \pi}\left(\ln \frac{m_{\tilde{q}}^{2}}{Q^{2}}+\frac{11}{12}\right),
$$

where we have assumed the scalar partners of the first two generations are degenerate with a mass $m_{\tilde{q}}$. If we further assume the initial condition, Eq. (1), then the size of the correction at $M(M)$ can be evaluated, as shown in Fig. 7 . It is clear that using the ratio $M_{a} / g_{a}^{2}$ (uncompensated for 
the superoblique corrections) implies corrections of order a few to tens of percent, depending on the gaugino involved and the separation of scales.

\section{Two-loop effects beyond the MSSM}

Throughout the previous section various effects were discussed that could change the evolution of $M_{a} / \alpha_{a}$ in the MSSM. Extensions of the MSSM could also easily give corrections to $M_{a} / \alpha_{a}$, usually through modifications to the two-loop $\beta$-function coefficients. In the following, two classes of well-motivated extensions of the MSSM will be explored. The first postulates an extra $U(1)^{\prime}$ symmetry, using both a "minimal" $U(1)^{\prime}$ model and an $E_{6}$ model as examples. The second extension postulates extra matter between the weak scale and the high scale.

\section{1 $\quad$ Extra $U(1)^{\prime}$}

The group structure of the MSSM can be easily extended to include an extra $U(1)$ group. There are many motivations for such an extension [48], such as a solution to the $\mu$-problem, string or GUT breaking to the MSSM plus an additional $U(1)$, etc. However, enlarging the group structure of the MSSM does not come without its own subtleties. The most obvious difficulty is to assign $U(1)^{\prime}$ charge such that all the anomalies are canceled; this is, in general, a difficult problem. In Appendix B we present the conditions on the multiplicities and charges of matter transforming under a $U(1)^{\prime}$ that cancel the anomalies. Although the $U(1)_{Y}$ anomaly conditions were not explicitly stated, cancellation is guaranteed if, for example, matter is added in complete representations of $S U(5)$ or is added in vectorlike pairs transforming under the MSSM gauge group. It was shown in Ref. [49] that a simple model can be constructed with $U(1)^{\prime}$ charges to one generation (only) of the MSSM matter that is nonanomalous, and this will serve as our "minimal" $U(1)$ example. We will also examine an $E_{6}$ model with three complete generations of $27 \mathrm{~s}$, which is also well known to be nonanomalous. We will not attempt to survey all possible extensions that include an extra $U(1)$, but instead consider mainly just these two classes of models. However, the particular breaking pattern of a GUT group, such as $E_{6}$, can change the results depending on the scale of the breaking and whether other group structures exist at intermediate scales. We will briefly comment on this in Sec. 4.2. Nevertheless, we expect that the results would not be significantly different if another $U(1)^{\prime}$ model ansatz were chosen that was valid up to near the high scale.

Before we present results for particular models, it is useful to understand the origin of corrections to the ratio $M_{a} / \alpha_{a}$ due to a $U(1)^{\prime}$ extension. First, the superpotential is assumed to have the term

$$
W \subset Y_{S} S H_{1} H_{2}
$$

in place of $\mu H_{1} H_{2}$, where the superfield $S$ is a gauge singlet under $S U(3)_{c} \times S U(2)_{L} \times U(1)_{Y}$. This in itself is a modification of the MSSM that could be considered separately, but comes with its own set of difficulties [50]. We will concentrate on a model with an extra $U(1)$ that will be broken 
when $S$ acquires a vacuum expectation value (vev). To avoid reintroducing the $\mu$-problem into these models, the term $\mu H_{1} H_{2}$ is assumed to be forbidden (by a judicious assignment of $U(1)^{\prime}$ charge). When $S$ acquires a vev, an effective $\mu=Y_{S}\langle S\rangle$ is generated, and thus the CP-odd Higgs boson and all neutralinos in the MSSM will acquire mass. The particle content of this minimal extension includes a $Z^{\prime}$, a new gaugino $\lambda_{S}^{\prime}$, a new Higgsino $\tilde{S}$, a scalar $S$, and a pseudoscalar. The pseudoscalar associated with $S$ is eaten by the $Z^{\prime}$, giving it mass via the Higgs mechanism. Three new soft terms are introduced

$$
-\mathcal{L}_{\text {soft }} \subset m_{S}^{2}|S|^{2}+\frac{1}{2}\left(M^{\prime} \lambda_{S}^{\prime} \lambda_{S}^{\prime}+\text { h.c. }\right)+\left(Y_{S} A_{S} S H_{1} H_{2}+\text { h.c. }\right)
$$

(we take $m_{S}, M^{\prime}$, and $A_{S}$ to be real) to give mass to the new gaugino and the uneaten scalar from the MSSM singlet superfield $S$, and a scalar trilinear coupling associated with the new Yukawa coupling. The neutralino mass matrix enlarges to $6 \times 6$, and the neutral CP-even Higgs mass matrix enlarges to $3 \times 3$. A new gauge coupling $g^{\prime}$ exists for the $U(1)^{\prime}$, giving two new couplings $\left(g^{\prime}\right.$ and $\left.Y_{S}\right)$ that enter the two-loop RG equations for the gauge couplings and gaugino masses. There is also a new one-loop invariant $M^{\prime} / g^{\prime 2}$. For a general set of $U(1)^{\prime}$ charges $Q_{i}$ assigned to the MSSM multiplets, we have computed the two-loop renormalization group equations for all the gauge couplings $\left(g_{1}, g_{2}, g_{3}, g^{\prime}\right)$ and gaugino masses $\left(M_{1}, M_{2}, M_{3}, M^{\prime}\right)$ including the effects of the additional Yukawa coupling $Y_{S}$. The results are presented in Appendix A.

Unlike the MSSM gauge and Yukawa couplings, the gauge coupling $g^{\prime}$ and the Yukawa coupling $Y_{S}$ are not determined by low energy experiments, and are therefore essentially free parameters. (Of course there will be constraints on these parameters in particular models from nonobservation data.) The size of the effects of the additional $U(1)^{\prime}$ are therefore highly model dependent. In practice, the two-loop corrections are not expected to be arbitrarily large unless $g^{\prime}$ or $Y_{S}$ approach a Landau pole near the high scale. However, we will only consider models in which $g^{\prime}$ and $Y_{S}$ can be treated perturbatively throughout the energy scale of interest, which will limit the size of the effect.

There is an additional parameter when two or more $U(1)$ groups are present in the low energy effective theory. The kinetic terms of $U(1)_{Y}$ can mix with those of $U(1)^{\prime}$ with a strength that is a priori unknown and a free parameter (again, in particular models there are constraints from experiment) [51, 52, 53, 54, 55]. Furthermore, the mixing is not RG invariant if

$$
\operatorname{tr}\left(Q_{a} Q_{b}\right) \neq 0
$$

the product of charges of the two groups, $U(1)_{a} \times U(1)_{b}$, summed over all chiral representations in the theory is nonzero. Although it would be interesting to know if there are consequences of kinetic mixing on the evolution of $M_{a} / \alpha_{a}$, we do not expect qualitatively different results, and therefore we do not consider this further in this paper. In particular, we will consider extra $U(1)$ models that obey $\operatorname{tr}(Y Q)=0$ and have their kinetic mixing set (by hand) to zero.

\footnotetext{
${ }^{4}$ When $g_{a}$ and $M_{a}$ are specified for a model with an additional $U(1)$, the $a=4$ elements correspond to $g^{\prime}$ and $M^{\prime}$.
} 


\subsection{Minimal $U(1)^{\prime}$ model}

One of the simplest extensions of the MSSM that includes an additional $U(1)^{\prime}$ was extensively studied in Ref. 49]. They proposed a superpotential with two Yukawa couplings,

$$
W=Y_{u} Q H_{2} u^{c}+Y_{S} S H_{1} H_{2},
$$

where the up-type Yukawa $Y_{u}$ couples only to the third generation, and the down-type Yukawas $Y_{d}$ and $Y_{e}$ are absent (actually forbidden by $U(1)^{\prime}$ charge assignments). The $U(1)^{\prime}$ charge assignments consistent with the cancellation of anomalies are given in Appendix B.2. The undetermined parameters of the model in addition to those in the MSSM are (in our conventions): the product of the $U(1)^{\prime}$ charge for $H_{1}$ and the $U(1)^{\prime}$ gauge coupling $Q_{1} g^{\prime}$, the $U(1)^{\prime}$ gaugino mass $M^{\prime}$, the Yukawa coupling $Y_{S}$, and the associated scalar trilinear coupling $A_{S}$.

Since $U(1)$ couplings are not asymptotically free, any additional $U(1)^{\prime}$ coupling added to the theory will have the greatest effect on the RG evolution of other parameters near the high scale. In general, it is hard to achieve any significant correction due solely to a larger $g^{\prime}$ without running to a Landau pole very near the high scale. In the following, we will take the initial condition in Eq. (1) , to hold at $1 \mathrm{TeV}$, analogous to the analyses done in Sec. 2. Furthermore, $Q_{1} g^{\prime}$ is set to $g_{1}\left(=g_{2}\right)$ at the high scale, $M^{\prime}=c M_{3}$ at the weak scale where $c$ is a constant, and for now Yukawa contributions to the two-loop RG equations are ignored. With these assumptions, the correction to $M_{a} / \alpha_{a}$ is less than $0.004 c$ after evolving 13 orders of magnitude in scale. Even if the coupling $Q_{1} g^{\prime}$ is increased to of order one, the correction is smaller than about 0.01c. Unless $M^{\prime}$ is taken to be considerably larger than $M_{3}$ (which would be disfavored by naturalness arguments), the correction due to "pure $U(1)^{\prime}$ gaugino" contributions to two-loop running is essentially negligible.

However, when the Yukawa couplings are nonzero, larger deviations can be obtained without soft parameters exceeding of order $M_{3}$ near the weak scale. In Fig. 8 we show the corrections to $M_{a} / \alpha_{a}$ arising from a model with $Y_{S}=1$ at the high scale, and $Y_{t}$ finite (we set $\tan \beta \approx 5$; lower or higher values of $\tan \beta$ give corrections analogous to Figs. 2 and 3). Again, in complete analogy to the MSSM studied in Sec. 2, large Yukawa couplings typically imply that the scalar trilinear couplings run to multi- $\mathrm{TeV}$ scale values near the high scale, which causes the nontrivial correction in $M_{a} / \alpha_{a}$. The size of the corrections are, in order from largest to smallest, $M_{2} / \alpha_{2}$, $M_{1} / \alpha_{1}, M_{4} / \alpha_{4}, M_{3} / \alpha_{3}$. The ratio $M_{3} / \alpha_{3}$ receives the smallest correction because the $\beta$-function coefficient $C_{3}^{S}=0$, and thus it is only through $Y_{S}$ feeding into $Y_{u}$ that there is any correction at all.

\section{$3.3 \quad \eta$-model of $E_{6}$}

There has been an enormous amount of work studying the phenomenology of superstring theories, particularly the group structures that emerge from compactification. One distinct possibility is $E_{6}$ [56, 57, 58, 59, 60, 61], which can arise from $E_{8}$ under suitable breaking. $E_{6}$ itself is rank-6, and one commonly studied breaking pattern is $E_{6} \rightarrow S O(10) \times U(1) \rightarrow S U(5) \times$

$U(1) \times U(1)$. Although the effects of two additional $U(1)$ 's could itself be a particularly interesting possibility to study, we restrict ourselves to considering the rank-5 $\eta$-model $S U(5) \times U(1)_{\eta}$, and 

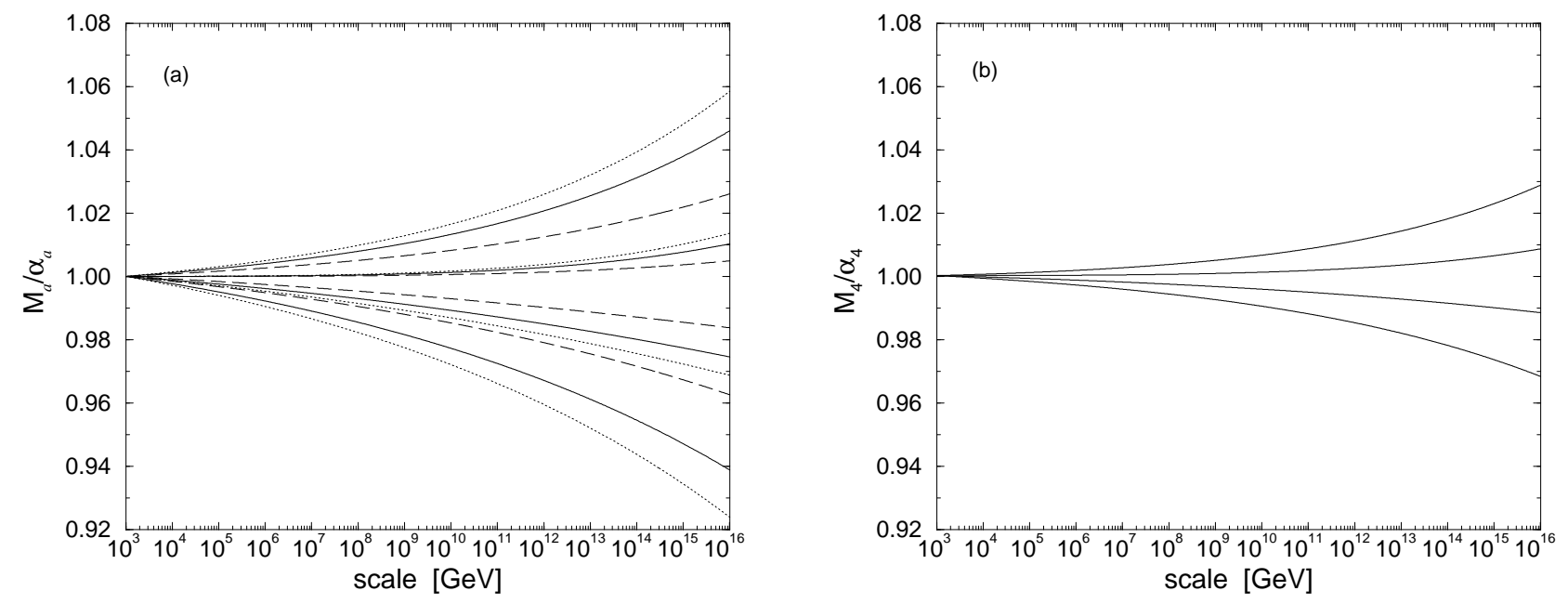

Figure 8: Evolution of $M_{a} / \alpha_{a}$ as a function of scale, for $Y_{S}\left(10^{16} \mathrm{GeV}\right)=1$, and $A_{t}=A_{S}=$ $M_{3}, 0,-M_{3},-2 M_{3}$ at $Q=1 \mathrm{TeV}$ from top to bottom for each triplet of lines in (a), and for each line in (b). The solid, dotted, and dashed lines correspond to $a=1,2,3$ respectively in (a), and the solid lines correspond to $a=4$ in (b). The ratio $M_{a} / \alpha_{a}$ has been normalized against an equivalent model with the Yukawa couplings set to zero.

further assume $S U(5)$ breaks near the scale where $E_{6}$ was broken. (Changing the scale where the GUT breaks, and other possible breaking patterns will be discussed in Sec. 4.2.) The $U(1)_{\eta}$ is a mixture of the two $U(1)$ 's in $E_{6}$ that is assumed to survive to near the weak scale. This provides a well-motivated example of a model with an extra $U(1)$ in which the charge assignments and overall normalization are determined (by embedding into $E_{6}$; see Table 2 for the charge assignments).

In particular, we consider an $\eta$-model with three generations of $\mathbf{2 7}$, which is the smallest number of representations that incorporates the MSSM and is nonanomalous. Unlike the minimal $U(1)^{\prime}$ model considered above in Sec. 3.2, all three generations are charged under the $U(1)^{\prime}$, and Yukawa couplings for $b$ and $\tau$ are present. Although the latter is not expected to affect the results of evolving the ratios $M_{a} / \alpha_{a}$ to the high scale (unless $\tan \beta$ is large), it does overcome a limitation of the minimal model. Three complete 27 representations of $E_{6}$ include three generations of the following: MSSM matter $\left(Q, u^{c}, d^{c}, L, e^{c}\right)$, down-type and up-type Higgs doublets $\left(H_{1}, H_{2}\right)$, two color triplets $\left(D, D^{c}\right)$, a right handed neutrino $\left(\nu^{c}\right)$, and a MSSM gauge singlet $(S)$. The superpotential for the model is

$$
W=Y_{t} Q H_{2} u^{c}+Y_{d} Q H_{1} d^{c}+Y_{e} L H_{1} e^{c}+Y_{S} S H_{1} H_{2}+Y_{D} S D D^{c} .
$$

Additional terms are possible, and indeed some are probably necessary for a phenomenologically viable model [62, 60]. However, additional dimension-3 terms in the superpotential with small Yukawa couplings will not affect the results presented below. To prevent large tree-level flavor

\footnotetext{
${ }^{5}$ The other $U(1)$ orthogonal to $U(1)_{\eta}$ is assumed to break at the high scale. In this section, we neglect effects of this high scale $U(1)$.
} 
changing neutral currents, only one set of Higgs $\left(H_{1}, H_{2}, S\right)$ is assumed to couple to the quark and lepton superfields. To break the $U(1)_{\eta}$, give mass to the color triplets, and generate an effective $\mu$ term, the scalar component of $S$ is assumed to acquire a vev. While the details of the vacuum structure (including avoiding possible charge/color breaking minima) are very important to be able to construct viable models of $E_{6}$, we will not discuss this further Our intention is to take the matter content of an $E_{6}$ model with the Yukawa couplings given in Eq. (22), and examine the consequences for the evolution of $M_{a} / \alpha_{a}$.

The RG equations for a three generation $E_{6}$ model are given in Appendix A, including the two-loop terms proportional to $Y_{S}^{2}$ and $Y_{D}^{2}$ in the gauge coupling and gaugino mass RG equations. It is well known that an $E_{6}$ model with three complete 27 s does not preserve gauge coupling unification, due to the extra Higgs doublets and color triplets. Although it is possible to add extra matter to the theory to bring the gauge couplings back into alignment at the high scale (see e.g. 63, 64, 53]), we defer a discussion of the effects of extra matter (beyond that needed to fill a $\mathbf{2 7}$ of $E_{6}$ ) to the next section.

There are four main effects that could alter the running of $M_{a} / \alpha_{a}$ in this model: the extra matter, the $U(1)_{\eta}$ gaugino mass, and the Yukawa couplings $Y_{S}$ and $Y_{D}$. The simplest way to illustrate the effect of the extra matter is to set the gauge coupling $g^{\prime}$ and the Yukawa couplings $Y_{S}$ and $Y_{D}$ to be small. Although some of the extra matter will obtain masses proportional to these Yukawa couplings, we will simply work in the approximation where a common scale $Q_{\text {extra }}$ can be chosen for the masses of the extra matter. By this we mean the two generations of pairs of Higgs doublets that do not couple to quarks and leptons (the "unHiggses"), all three generations of the color triplets, the right-handed neutrinos, and the MSSM gauge singlets. In Fig. 9(a), we show the evolution of $M_{a} / \alpha_{a}$ in a model where, again, the initial condition in Eq. (11) is assumed. The ratios $M_{a} / \alpha_{a}$ are normalized against an equivalent model with $Q_{\text {extra }}>M_{\text {unif }}$; the latter is equivalent to a model without the $E_{6}$ matter content (at the weak scale). Since only the extra matter can alter the evolution (when $g^{\prime}, Y_{S}, Y_{D}$ are small), Fig. 9(a) shows that the three generations of color triplets induce the largest correction manifested in $M_{3} / \alpha_{3}$, although sizeable corrections are also present for $M_{1} / \alpha_{1}, M_{2} / \alpha_{2}$. The gauge couplings $g_{1}$ and $g_{2}$ are nearly $30 \%$ larger at】 $M_{\text {unif }} \sim 3 \times 10^{15} \mathrm{GeV}$, while $g_{3}$ is nearly $70 \%$ larger, as compared to a model without the extra matter. In practice this means that the effects of the additional $U(1)_{\eta}$ coupling $g^{\prime}$ and the Yukawa couplings $Y_{S}, Y_{D}$ are not nearly as significant to the RG evolution of $M_{a} / \alpha_{a}$, since they compete against the considerably larger gauge couplings. If we include the effects of the $U(1)_{\eta}$ gaugino, the shift in the curves of Fig. 9(a) is at the level of about 0.007c, where $M^{\prime}=c M_{3}$ at the weak scale. Including Yukawa couplings $Y_{S}\left(M_{\text {unif }}\right) \approx Y_{D}\left(M_{\text {unif }}\right) \approx 1$, with scalar trilinear couplings in the range $-2 M_{3} \leq A_{x} \leq M_{3}$, there are more significant effects, as shown in Fig. 9(b). Again, the combined effects of large gauge couplings with larger Yukawa couplings push the scalar trilinear couplings to multi-TeV values at $M_{\text {unif }}$.

Up to now we have tentatively placed the extra matter at $Q_{\text {extra }}=1 \mathrm{TeV}$. If the scale of the extra matter were increased to $2,10 \mathrm{TeV}$, one finds the shift in $M_{a} / \alpha_{a}$ at $Q=M_{\text {unif }}$ is roughly

\footnotetext{
${ }^{6}$ The mapping of our results onto completely viable weak scale models derived from $E_{6}$ is beyond the scope of this paper.

${ }^{7} M_{\text {unif }}$ was somewhat arbitrarily chosen to be the where $g_{1}$ and $g_{2}$ meet, which is lower in this $E_{6}$ model.
} 

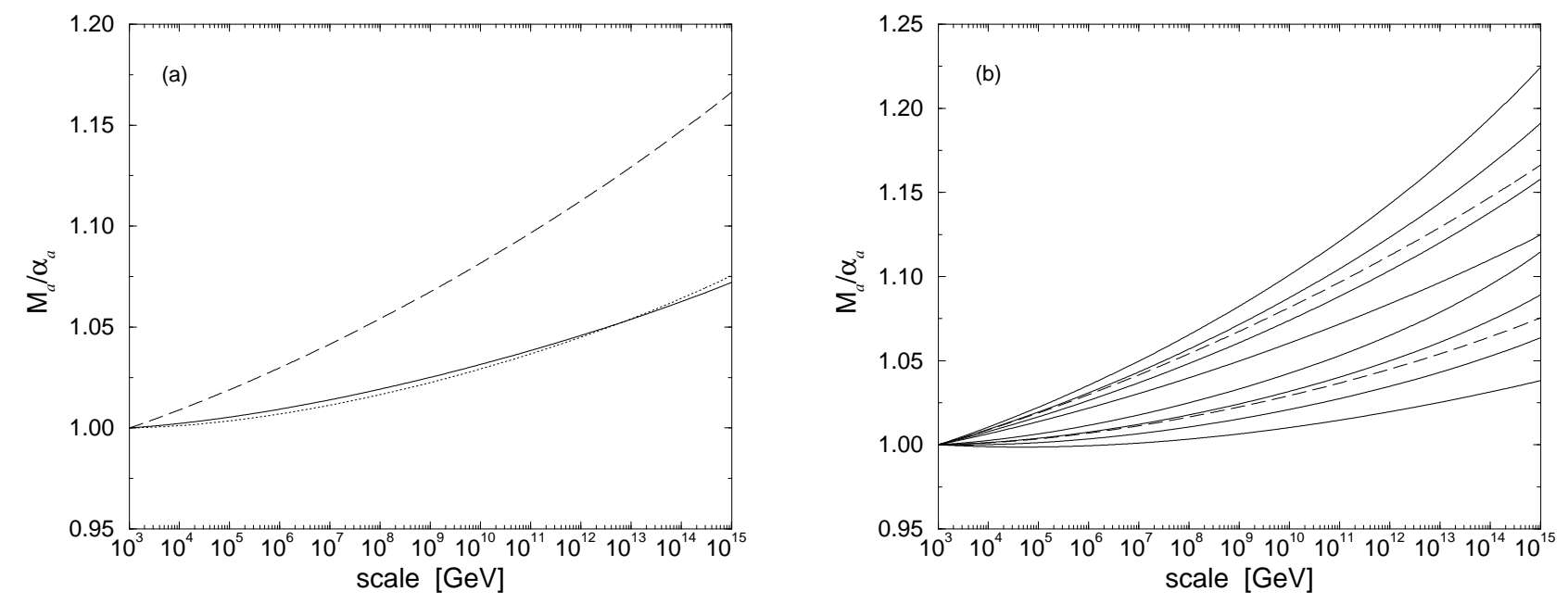

Figure 9: Evolution of $M_{a} / \alpha_{a}$ as a function of scale for an $E_{6}$ model with (a) the Yukawa couplings $Y_{S}$ and $Y_{D}$ set to zero, and (b) the Yukawa couplings $Y_{S}\left(M_{\text {unif }}\right) \approx Y_{D}\left(M_{\text {unif }}\right) \approx 1$. In graph (a) the solid, dotted, and dashed lines correspond to $a=1,2,3$. In graph (b) the four solid lines in the upper and lower part of the graph correspond to $A_{x}=M_{3}, 0,-M_{3},-2 M_{3}$ at $Q=1 \mathrm{TeV}$ for $M_{3} / \alpha_{3}$ and $M_{2} / \alpha_{2}$ respectively. For comparison we also plotted $M_{3} / \alpha_{3}$ and $M_{2} / \alpha_{2}$ from Fig. 9(a) as dashed lines. The ratios have been normalized against a model without the extra matter needed to fill complete 27 representations of $E_{6}$.

$(-0.002,-0.002,-0.011),(-0.019,-0.019,-0.046)$ for $a=(1,2,3)$ respectively, relative to the results in Fig. 9(a). Hence, increasing the scale of the extra matter by even a small amount (one order of magnitude of twelve), reduces the correction to $M_{a} / \alpha_{a}$ by several percent.

\subsection{Extra matter}

In the $\eta$-model of $E_{6}$ described above, it was necessary to introduce extra matter to fill complete 27s to ensure the cancellation of extra $U(1)$ anomalies. Indeed, the extra matter dominated the correction to $M_{a} / \alpha_{a}$. However, extra matter can, of course, be added without enlarging the gauge structure of the model or even disrupting gauge coupling unification, as long as anomaly cancellation with the MSSM group is ensured. A recent, widely touted example of adding extra matter without upsetting gauge-coupling unification is the addition of $\mathbf{5}+\overline{\mathbf{5}}$ pairs (or a $\mathbf{1 0}+\overline{\mathbf{1 0}}$ pair) used as the messenger sector of gauge-mediated models [65, 66]. While we defer going into details about gauge-mediation (particularly the supersymmetry breaking masses associated with the messenger matter) until Sec. 5, this does serve as one interesting starting point for adding extra matter. Nevertheless, for the remainder of the section the extra matter is assumed to have only supersymmetry preserving masses.

It is perhaps useful to review a few recent examples of the uses of extra matter for modelbuilding and phenomenology (other than gauge-mediation). Ref. [67] showed that adding extra matter could "refocus" the gauge couplings to unify at a higher scale. The motivation was 
an attempt to bridge the discrepancy between $M_{\text {unif }}$ and $M_{\text {string, }}$, and indeed they were able to show that only particular kinds of extra matter were able to do the job. In Ref. [28] a "semi-perturbative" model was constructed whereby the gauge couplings became large, but still perturbatively calculable, at the high scale. They added sufficient $\mathbf{5}+\overline{\mathbf{5}}$ and $\mathbf{1 0}+\overline{\mathbf{1 0}}$ pairs to nearly saturate the $\beta$-functions, and found the interesting result that the gaugino mass ratios $M_{a} / M_{b}$ at the weak scale could be quite different from the canonical expectations of models without the extra matter. By adding matter in complete representations of $S U(5)$, gauge-coupling unification (to one-loop) is not upset. However, as pointed out in Ref. [68, extra matter can be added in other combinations that also maintain (one-loop) gauge coupling unification, indicating that there are a variety of possibilities of extra matter that could be explored. Finally, Ref. 69 showed that adding extra matter in complete $\mathbf{5}+\overline{\mathbf{5}}$ and $\mathbf{1 0}+\overline{\mathbf{1 0}}$ does not change 8 the $\alpha_{s}$ value extracted at the weak-scale, but could increase the unification scale. In particular, by taking the heavy multiplets to have a common mass at the high scale but split in mass at intermediate scales (by RG gauge corrections), they showed that the one-loop threshold corrections largely cancel the two-loop corrections in the $\beta$-functions. Although this is important to determine (separately) the gauge couplings or gaugino masses at the high scale, the one-loop threshold corrections cancel in the ratio $M / \alpha$. Thus, in the examples to follow, we will generally assume the extra matter is introduced with a common mass at a single intermediate scale.

Extra matter can be safely added in vectorlike pairs, allowing an arbitrarily large supersymmetric mass term and ensuring the cancellation of anomalies. We therefore have two sets of parameters; the quantity (and type) of extra matter, and the scale(s) where it is introduced. Using the results in Appendix A, extra matter can be easily incorporated by merely shifting the $\beta$-function coefficients at the scale of the new matter, creating a new effective theory. Increasing the amount of extra matter can be compensated by increasing the scale where the matter is introduced, and so following Ref. [28] we introduce an effective amount of extra matter $n_{\text {eff }}$ that (in particular) need not be an integer. In this way, extra matter need only be added at one scale (that we take to be near the weak scale $\sim 1 \mathrm{TeV}$ ), and then the size of the deviation in $M_{a} / \alpha_{a}$ is computed as a function of the continuous parameter $n_{\text {eff }}$.

The type of extra matter introduced could be of many different varieties, however there are constraints. If we restrict ourselves to vectorlike matter, then there could be vectorlike pairs of MSSM matter (e.g. $Q+\bar{Q}$ ), or limitless varieties of exotics that may (or may not) fill representations of common GUT groups, and that may (or may not) preserve gauge coupling unification. We will consider only four scenarios of adding extra matter, and we decided to examine scenarios that do not upset gauge coupling unification (at least to one-loop). The scenarios are:

$$
\begin{array}{ll}
\text { Set 1: } & n_{\mathrm{eff}}=n_{\mathbf{5}}+n_{\overline{\mathbf{5}}} \\
\text { Set 2: } & n_{\mathrm{eff}}=n_{\mathbf{1 0}}+n_{\overline{\mathbf{1 0}}} \\
\text { Set 3: } & n_{\mathrm{eff}}=2 n_{Q}=2 n_{d}=4 n_{e} \\
\text { Set 4: } & n_{\mathrm{eff}}=2 n_{Q}=4 n_{u}=2 n_{L} .
\end{array}
$$

\footnotetext{
${ }^{8}$ With up to intermediate values of the gauge couplings at the unification scale. For much larger values the expectations are quite different [28, 70].
} 

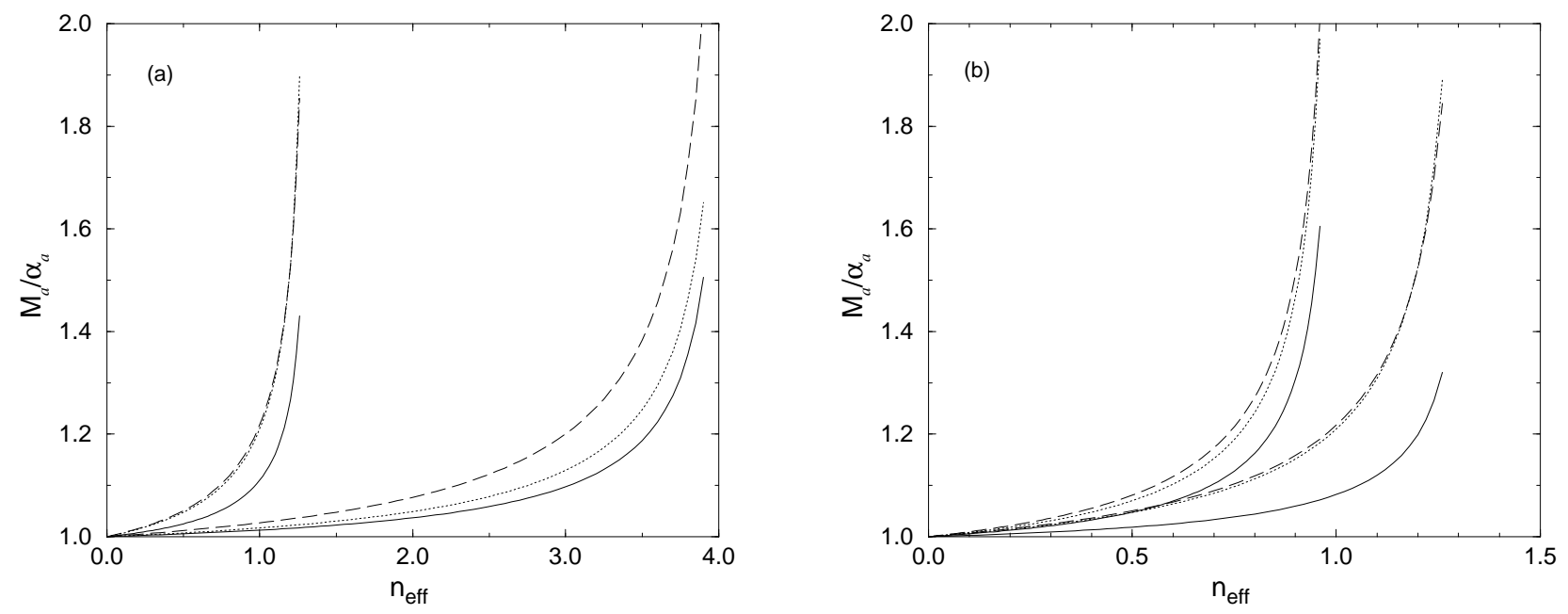

Figure 10: $M_{a} / \alpha_{a}$ evaluated at $Q=10^{16} \mathrm{GeV}$ with four classes of extra matter, normalized to a model without extra matter. The solid, dotted, and dashed lines correspond to $a=1,2,3$.

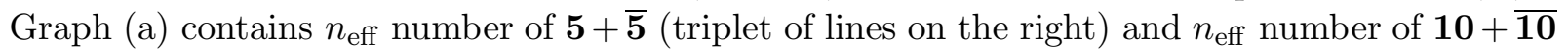
(triplet of lines on the left), and graph (b) contains $n_{\text {eff }}$ number of Set 3 (triplet of lines on the right) and $n_{\text {eff }}$ number of Set 4 (triplet of lines on the left).

Set 1 and Set 2 are the common extensions of adding vectorlike matter in complete $S U(5)$ representations $\mathbf{5}+\overline{\mathbf{5}}$ and $\mathbf{1 0}+\overline{\mathbf{1 0}}$ pairs, respectively. We also consider adding

$$
\text { Set } 3: \quad\left(\mathbf{3}, \mathbf{2}, \frac{1}{6}\right)+\left(\overline{\mathbf{3}}, \mathbf{1}, \frac{1}{3}\right)+2 \times(\mathbf{1}, \mathbf{1}, 1)+\text { conj } .
$$

and

$$
\text { Set } 4: \quad\left(\mathbf{3}, \mathbf{2}, \frac{1}{6}\right)+2 \times\left(\overline{\mathbf{3}}, \mathbf{1},-\frac{2}{3}\right)+\left(\mathbf{1}, \mathbf{2},-\frac{1}{2}\right)+\text { conj. }
$$

which do not apparently form complete representations of any simple GUT group (Set 3 was first considered in Ref. [68]). Nevertheless, Sets 3 and 4 ensure the one-loop $\beta$-functions are shifted by the same amount independent of the particular coupling, and therefore one-loop gauge coupling unification is preserved.

In Fig. 10 we show the effect of adding $n_{\text {eff }}$ number of pairs of the additional matter in one of the above scenarios. The curves are cut off when any coupling $\alpha$ exceeds the somewhat arbitrary value 0.5 ; beyond this value, perturbative calculations are probably not reliable [28]. It is clear that the size of the correction to $M_{a} / \alpha_{a}$ by adding extra matter can be very large, in comparison to all the previous effects that have been considered. However, this occurs only when the gauge couplings are near their semi-perturbative value, and indeed we were unable to find corrections much larger than $20 \%$ to $30 \%$ without a gauge coupling exceeding $\alpha \sim 0.2$ at $10^{16} \mathrm{GeV}$. In all cases above, the largest correction occurred for $M_{3} / \alpha_{3}$ due to our choice of adding extra matter that preserves one-loop gauge coupling unification, that implies $g_{3}$ remains the largest gauge coupling throughout the RG evolution. 
The effect of increasing the mass $M_{\text {extra }}$ of the extra matter can be easily approximated by rescaling $n_{\text {eff }}$ by a factor

$$
n_{\mathrm{eff}} \rightarrow \frac{\ln \frac{Q_{\mathrm{high}}}{1 \mathrm{TeV}}}{\ln \frac{Q_{\mathrm{high}}}{M_{\text {extra }}}} n_{\mathrm{eff}}
$$

where $Q_{\text {high }} \sim 10^{16} \mathrm{GeV}$. If the amount of running is reduced $Q_{\text {high }}<10^{16} \mathrm{GeV}$, the rescaling of $n_{\text {eff }}$ is more complicated because the three gauge couplings are no longer expected to be equal at the scale where the running is stopped.

\section{High scale corrections}

There are a variety of effects that can upset the ratio $M_{a} / \alpha_{a}$ near the high scale. In the following, we will examine the well-known effects of GUT scale threshold corrections [71], discuss issues related to the scale of GUT breaking, examine Planck scale corrections, and finally discuss effects unique to supergravity.

\subsection{GUT scale thresholds}

If gauge coupling unification is indeed indicative of a grand unified theory, then many additional heavy fields near the high scale will be present. In $S U(5)$, for example, there are the heavy gauge boson and gaugino remnants of the adjoint, transforming as $\left(\mathbf{3}, \mathbf{2}, \frac{5}{6}\right)$ under $S U(3)_{c} \times S U(2)_{L} \times U(1)_{Y}$, as well as heavy Higgs multiplets (from, e.g. the $\mathbf{2 4}$ and $\mathbf{5}+\overline{\mathbf{5}}$ of Higgs), and possibly heavy chiral multiplets as well. The corrections to the ratio $M_{a} / \alpha_{a}$ due to integrating out the heavy remnants of GUT breaking were calculated in Ref. [71], and we will follow their discussion closely. There are two classes of corrections to the ratio $M_{a} / \alpha_{a}$ : wave function renormalization and mass renormalization. For a heavy chiral or vector supermultiplet with a mass $M_{\text {heavy }}$, the wave function renormalization corrections give rise to logarithmic corrections to $M_{a}$ and $g_{a}$ that scale as

$$
\sim \frac{\alpha}{4 \pi} \ln \frac{M_{\text {heavy }}^{2}}{Q_{\mathrm{GUT}}^{2}},
$$

after integrating out the heavy fields near the GUT scale. Of course this is completely equivalent to modifying the $\beta$-function coefficients at the scale of the heavy fields, and therefore the logarithmic corrections cancel in the ratio $M_{a} / \alpha_{a}$ [71, 28] to one-loop. (Two-loop corrections can be neglected since we are assuming the heavy fields are near the GUT scale, and so no large logarithms are present.)

The second class of corrections arise when soft supersymmetry breaking masses are introduced in the mass matrices for the heavy fields, leading to mass renormalization. In a general supersymmetric theory with soft breaking, we would expect soft terms for all sparticles with a scale of order the breaking scale $M_{\mathrm{SUSY}}$, presumably near the weak scale. The soft breaking 
masses are ordinarily insignificant compared with the supersymmetry preserving masses induced from GUT breaking. However, the corrections to $M_{a} / \alpha_{a}$ take the form [72]

$$
\frac{M_{a}}{\alpha_{a}}=\frac{M_{5}\left(Q_{\mathrm{GUT}}\right)}{\alpha_{5}\left(Q_{\mathrm{GUT}}\right)}+\frac{1}{4 \pi}\left(2 C\left(G_{a}\right)\left[M_{5}\left(Q_{\mathrm{GUT}}\right)-\delta m\right]+\sum_{R} S_{a}(R) B_{R}\right)
$$

where $M_{5}\left(Q_{\mathrm{GUT}}\right)$ and $\alpha_{5}\left(Q_{\mathrm{GUT}}\right)$ are the unified gaugino mass and gauge coupling at the GUT scale $Q_{\mathrm{GUT}}, \delta m$ is the mass of the fermion component of the Nambu-Goldstone multiplet induced by supersymmetry breaking, and $B_{R}$ are the standard $B$-terms in the soft supersymmetry breaking Lagrangian for the chiral representations $R$. The group theory factors are the quadratic Casimir $C\left(G_{a}\right)$ for the adjoint $G_{a}$, and the Dynkin index $S_{a}(R)$ for the chiral representation $R$, both for the group associated with the " $a$ " gaugino. When the supersymmetry breaking masses $M_{5}, \delta m$ and $B_{R}$ are roughly the same scale as the gaugino masses, the correction is typically of order a few percent.

In the case where the GUT scale chiral multiplets fill up a complete representation of the GUT group, the corrections $\sum_{a} S_{a}(R) B_{R}$ are finite but universal. This is completely analogous to integrating out the messenger sector of gauge-mediated models, and indeed the calculation of the gaugino mass in gauge-mediated models [65, 68] is identical to the calculation above, in the limit that the mass splitting of the scalar components of the messenger superfields is much smaller than the associated fermion mass. The presence of heavy fields with supersymmetry breaking masses is a bona fide possibility in itself, and the heavy fields need not be associated with a GUT nor do they need to have masses near the GUT scale (although a GUT does provide a strong motivation for the existence of such fields). Thus GUT scale corrections to $M_{a} / \alpha_{a}$ are indeed possible, but the size of the effect depends solely on the size of the supersymmetry breaking masses associated with the heavy fields.

\subsection{Breaking GUTs at other scales}

When we discussed the $E_{6}$ GUT in Sec. 3.3, it was assumed that the breaking pattern $E_{6} \rightarrow S U(5) \times U(1) \times U(1) \rightarrow S U(3)_{c} \times S U(2)_{L} \times U(1)_{Y} \times U(1)_{\eta}$ occurred near the high scale. Extrapolating $M_{a} / \alpha_{a}$ (for $1 \leq a \leq 4$ ) was therefore well-defined up to this GUT breaking scale. However, it is very possible that the breaking of the GUT group does not go directly to the standard model (plus extra $U(1)$ 's), but instead occurs in stages. For example, the rank-6 group $E_{6}$ could break to either $S O(10) \times U(1)$ or $[S U(3)]^{3}$, the latter breaking to $S U(3)_{c} \times$ $S U(2)_{L} \times S U(2)_{R} \times U(1)_{B-L}$ and then to the MSSM group. The rank-5 group $S O(10)$ could break to $S U(5) \times U(1)$ or $S U(4)_{c} \times S U(2)_{L} \times S U(2)_{R}$, and then there are various permutations of breaking the latter $S U(4)_{c}$ and $S U(2)_{R}$. The phenomenology of these breaking patterns has been well-studied (for a review, see Ref. [14]), and for our purposes it is important to recognize that the ratios $M_{a} / \alpha_{a}$ may only have meaning up to some intermediate scale, where part or all of the MSSM group is embedded in a more complicated (possibly higher rank) structure. It was pointed out in Ref. [73] that despite the possibly complicated breaking patterns in $S O(10)$, the expectations for the gaugino mass ratios at the weak scale to one-loop were identical to the case where the breaking is directly to the MSSM group. Two-loop corrections are probably 
small, unless the gauge or Yukawa couplings become large, or unless the two-loop coefficients are significantly altered by the extra matter in higher dimensional representations needed to accomplish the various stages of breaking. Above the breaking scale, the ratios $M_{a} / \alpha_{a}$ are subsumed into "new" one-loop invariants $M / \alpha$ associated with the group at that scale [e.g. $M_{5} / \alpha_{5}$ for $S U(5)$ or $M_{10} / \alpha_{10}$ for $\left.S O(10)\right]$. Although we have not attempted to analyze the various (two-loop and other) effects that could disrupt these GUT one-loop invariants, there would be nonzero corrections.

\subsection{Planck scale corrections}

It is well known that physics near the Planck scale can affect low energy predictions of, for example, the gauge couplings through higher dimension operators [74, 75]. These analyses presume quantum gravity induces nonrenormalizable (dimension $>4$ ) terms suppressed by the Planck scale? that can take the form [74]

$$
\frac{1}{M}\left[\operatorname{tr}\left(W^{\alpha} W_{\alpha} \Sigma\right)+h . c .\right]_{F},
$$

where $W^{\alpha}$ and $\Sigma$ are gauge and Higgs superfields and the trace is over the gauge group generators. If $\Sigma$ acquires a vev for its scalar component $S$ (through, e.g., the breaking of a GUT gauge group), then the kinetic terms for the gauge fields are modified

$$
\delta \mathcal{L}_{\text {kinetic }}=-\frac{1}{4 M} \operatorname{tr}\left(F_{\mu \nu} F^{\mu \nu} S\right)
$$

where $F_{\mu \nu} \equiv F_{\mu \nu_{a}} T^{a}$ is the field strength tensor of the unified gauge group. To bring the kinetic terms back into a canonical form, the fields are rescaled, and therefore the gauge couplings must also be rescaled. This is the well-known Planck scale correction to the gauge couplings, that has been discussed in Refs. [74, 75, 5, 76, 77, 78.

The Planck scale operator not only gives modifications to the gauge kinetic terms, but also modifies the gaugino kinetic terms (and auxiliary fields) [74, 79, 80]

$$
\delta \mathcal{L}_{\text {kinetic }}=\frac{1}{M} \operatorname{tr}\left(i \lambda \not \supset \bar{\lambda} S+\frac{1}{2} D^{2} S\right)
$$

where $\lambda$ is the 2-component gaugino field, $D$ is the auxiliary field, and $\mathcal{D}_{\mu}$ is the covariant derivative. To bring these terms back into a canonical form, the gaugino (and auxiliary) fields must also be rescaled. Hence, the soft term $\frac{1}{2} M(\lambda \lambda+h . c$. $)$ is rescaled, giving a correction to the gaugino mass simultaneously with the correction to the gauge coupling. It is simple to show that the correction to $M$ cancels in the ratio with $g^{2}$, and therefore Planck scale operators of the form in Eq. (32) do not affect the ratio $M / \alpha$. This is easily generalized to $M_{a} / \alpha_{a}$. Furthermore, any rescaling of the gauge kinetic terms (from higher dimension operators, or any other source) must be accompanied by rescaling of the gaugino kinetic terms by the same amount and therefore cancels in the ratio $M / \alpha$, if supersymmetry is to be preserved.

\footnotetext{
${ }^{9}$ Normally taken to be the "reduced" Planck scale $M=M_{P l} / \sqrt{8 \pi} \approx 2.4 \times 10^{18} \mathrm{GeV}$.
} 


\subsection{Supergravity effects}

In the previous section concerning Planck scale corrections of the type in Eq. (32), the operator was implicitly assumed to be globally supersymmetric. When this is generalized to a locally supersymmetric theory (supergravity), the Lagrangian becomes more complicated [81]. Specifically, the gauge kinetic terms plus gaugino soft terms take the form

$$
\mathcal{L} \subset-\frac{1}{4} \operatorname{Re} f_{a b} F_{\mu \nu}^{a} F^{\mu \nu b}-\frac{1}{4} \operatorname{Re} f_{a b} \bar{\Lambda}^{a} \not \supset \Lambda^{b}+\frac{1}{4} e^{-G / 2} G^{i}\left(G^{-1}\right)_{i}^{j} \frac{\partial f_{a b}^{*}}{\partial \phi^{j}} \bar{\Lambda}^{a} \Lambda^{b}
$$

where two new functions are introduced; the gauge kinetic function $f_{a b}$, and the Kähler potential $G$, with derivatives denoted by $G^{i} \equiv \partial G / \partial \phi_{i}$ and $G_{i}^{j} \equiv \partial^{2} G / \partial \phi^{* i} \partial \phi_{j}$. ( $\Lambda$ is the 4-component Majorana gaugino field and $\phi_{i}$ is the scalar component of the chiral superfields $\Phi_{i}$ in the theory.) The gauge kinetic function $f_{a b}$ can be thought of as parameterizing the dimension $>4$ Planck scale operators. The Kähler potential is indigenous to supergravity, and is a (real) function of the scalar components of the chiral superfields in the theory. Since its form is a priori unknown, the coefficient of the gaugino soft mass term is unknown. Scalar fields with vevs near the Planck scale are expected to modify the Kähler potential, and therefore modify the gaugino masses independent of the kinetic terms [79, 80, 78]. Thus, the relation $M_{a} / \alpha_{a}$ is expected to be modified when embedded in a supergravity theory with a non-minimal Kähler potential. This should not be surprising, since universal nonzero gaugino masses are accomplished by specifying $f_{a b}=S \delta_{a b}$

and $G_{i}^{j}=-\delta_{i}^{j}$ (a flat Kähler manifold), and determined by the gravitino mass proportional to $\exp (-\langle G\rangle / 2)$. More detailed computations of the gaugino masses in stringy supergravity models were carried out in Ref. 82] to which we refer the interested reader.

\section{$5 \quad$ Gauge-mediated masses}

The final class of "corrections" to $M_{a} / \alpha_{a}$ that we will consider is from extra matter with supersymmetry breaking masses. Ordinarily supersymmetry breaking is assumed to occur in a "hidden" sector, which is assumed to contain matter with no MSSM gauge charges. Consequently, only gravitational interactions exist between the hidden sector and the MSSM, and it is through gravity that supersymmetry breaking is communicated to the "visible" sector; this is the canonical "supergravity-inspired" model. An alternative possibility is that supersymmetry is broken in a sector that does have couplings to the MSSM, and thus ordinary gauge interactions can communicate supersymmetry breaking to the MSSM. This scenario has been the study of an enormous amount of recent work 65, 66.

In gauge-mediated models, a "messenger" sector is introduced that has both supersymmetry preserving masses and supersymmetry breaking masses. The supersymmetry preserving masses ensure that the messenger masses are considerably larger than the weak scale, while the supersymmetry breaking masses can successfully induce weak scale masses in the MSSM sparticles. The canonical approach [65] is to assume the messengers form complete representations of $S U(5)$ to ensure gauge coupling unification is not disrupted at one-loop (however, see Ref. [68]). The 


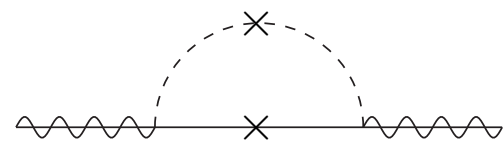

Figure 11: The one-loop gaugino mass induced by heavy messengers. The crosses represent the relevant mass insertions.

superpotential for the messenger sector takes the form

$$
W=\lambda S \Phi \bar{\Phi}
$$

where $\Phi, \bar{\Phi}$ transform as a vectorlike representation of the MSSM gauge group, such as

$$
\left(\mathbf{3}, \mathbf{1},-\frac{1}{3}\right)+\left(\mathbf{1}, \mathbf{2}, \frac{1}{2}\right)+\operatorname{conj} .
$$

that make up the $\mathbf{5}+\overline{\mathbf{5}}$ of $S U(5)$. Supersymmetry breaking occurs when the auxiliary component of the gauge singlet $S$ acquires a vev $F_{S}$, but we also assume the scalar component of $S$ also acquires a vev $\langle S\rangle$ to give $\Phi$ and $\bar{\Phi}$ (large) supersymmetry preserving masses. The spin-1/2 components of $\Phi$ and $\bar{\Phi}$ are combined as one single Dirac fermion, with a mass $m_{f} \equiv \lambda\langle S\rangle$. The two complex scalars from $\Phi$ and $\bar{\Phi}$ acquire masses $m_{1}$ and $m_{2}$, with $m_{1,2}^{2}=m_{f}^{2} \mp \lambda F_{S}$. In general, additional (higher dimensional) interactions could be added to Eq. (36); the effect of such terms is to modify the relation between $m_{1,2}$ and $m_{f}$, parameterized by Str $M_{\text {mess }}^{2} \equiv 2 m_{1}^{2}+2 m_{2}^{2}-4 m_{f}^{2}$ [83].

The one-loop messenger contributions to the gaugino masses have been presented in Refs. 65, [68], and generalized to the case $\operatorname{Str} M_{\text {mess }}^{2} \neq 0$ in Ref. [83]. The relevant diagram is shown in Fig. 11, which is equivalent to the mass renormalization diagram due to heavy chiral multiplets discussed in Sec. 4.1, and is the supersymmetry breaking analog to Fig. E(a). The result for the self-energy can be written as

$$
\Sigma(\not p)=\frac{\alpha}{4 \pi} m_{f} S(\Phi) \sin 2 \theta \times\left[\frac{y_{2}}{1-y_{2}} \ln y_{2}-\frac{y_{1}}{1-y_{1}} \ln y_{1}\right]
$$

where $y_{i}=m_{i}^{2} /\left(p^{2}+m_{f}^{2}\right), m_{f}$ is the messenger fermion mass, $m_{1}$ and $m_{2}$ are the messenger scalar masses, $\theta$ is the angle that diagonalizes the mass matrix $\mathbb{D}$, and $S(\Phi)$ is the usual Dynkin index for the messenger superfield $\Phi$. The correction to the gaugino mass can be approximated as simply

$$
M=M_{0}+\Sigma\left(M_{0}\right)
$$

where $M$ and $M_{0}$ is the gaugino mass with and without the supersymmetry breaking sectors integrated out. The expression given in Eqs. (38), (39) is a trivial generalization of the formulae given in Refs. [68, 83], and reduces to the latter formulae in the limit that the gaugino mass is induced entirely from messenger interactions. In fact, the $p^{2}$ correction in the denominator of $y_{i}$ is negligible when the messenger fermion mass is much larger than the gaugino mass, which is the usual situation.

\footnotetext{
${ }^{10}$ We assume the scalar mass matrix is real.
} 
The size of the correction to the gaugino mass is dependent entirely on the size of the supersymmetry breaking mass $\sqrt{F_{S}}$ in the messenger sector. In the case of heavy chiral multiplets with supersymmetry breaking masses of order the weak scale [71], the correction is of order a few percent. This is because the one-loop self-energy correction $\Sigma\left(M_{0}\right)$ is suppressed by $\alpha /(4 \pi)$. In gauge-mediated models, this suppression is overcome by specifying a supersymmetry breaking mass of order the weak scale times $4 \pi / \alpha$, which is of order tens of TeV. Note that two-loop corrections to the gaugino masses have also been recently computed in Ref. [84, and can give corrections up to $10 \%$ to the one-loop result if the messenger scale is of order the supersymmetry breaking mass.

It is also worth considering the possibility that extra heavy vectorlike matter exists with supersymmetry breaking masses 1 that do not come in complete representations of $S U(5)$, nor even preserve gauge-coupling unification. This class of matter would communicate its supersymmetry breaking to only those sparticles charged under the appropriate groups to which the extra matter transforms nontrivially. There are, however, some important comments. The size of the contribution to any given gaugino mass (and thus the ratio $M_{a} / \alpha_{a}$ ) is determined by the size of the supersymmetry breaking mass (and the multiplicity of the extra matter). The correction to the gauge couplings (at the high scale) due to the extra matter is determined solely by the multiplicity and the scale of the extra matter, i.e. the supersymmetry preserving masses $\square$. Thus, some classes (or multiplicities) of extra matter could be excluded if the gauge couplings are required to be perturbative to the high scale. The size of the correction to the gaugino mass is, however, undetermined. Hence, the correction to $M_{a} / \alpha_{a}$ could be arbitrarily large.

There are, of course, phenomenological consequences if supersymmetry is broken at scales smaller than the canonical scale $\sim 10^{11} \mathrm{GeV}$ assumed in supergravity models. In particular, a light gravitino is expected. The presence or absence of a light gravitino in collider experiments would help determine (or at least put a lower bound on) the fundamental scale of supersymmetry breaking (see e.g. 85, 86]). However, it probably does not determine the supersymmetry breaking masses in the extra (messenger) matter because generically one expects complicated feed down mechanisms in the dynamical breaking sectors that ultimately separate the "true" scale $\sqrt{F}$ from $\sqrt{F_{S}} 65$.

\section{Conclusions}

In this paper, we have studied the effects that can disrupt the one-loop renormalization group invariant, $M_{a} / \alpha_{a}$. This includes two-loop corrections in the MSSM, weak scale threshold corrections, and superoblique corrections. Two-loop corrections from extensions of the MSSM within a minimal $U(1)$ model, an $E_{6}$ model, and extra matter were also considered. In addition, high scale corrections from GUT thresholds, Planck scale operators, and supergravity effects

\footnotetext{
${ }^{11}$ To avoid the introduction of additional complications, the supersymmetry breaking can be assumed to be introduced explicitly. Those who find this unpalatable can imagine gauge singlet interactions (plus possibly higher dimensional terms) analogous to Eq. (36).

${ }^{12}$ Highly split supermultiplets will have additional corrections dependent on the size of the supersymmetry breaking masses.
} 
were discussed. Finally, the effects from a heavy sector with supersymmetry breaking masses that communicates via gauge-mediation to the MSSM were discussed.

The two-loop corrections (both within the MSSM, and beyond) were, not surprisingly, found to be large only when a coupling becomes large near the high scale. Otherwise, the corrections are of order a few to tens of percent after evolving 13 orders of magnitude in scale. The "pure gaugino" terms were usually small except for the $g_{3}^{2} M_{3}$ term, and in particular the extra $U(1)^{\prime}$ gaugino in extended models typically gives a very small correction. The Yukawa terms can induce a larger correction (up to ten to perhaps tens of percent at the high scale), particularly if $\tan \beta$ is near one of its perturbative extremes. Typically, to obtain a larger correction the scalar trilinear couplings must run to several times their weak scale values at the high scale. The Yukawa terms, and hence the correction to $M_{a} / \alpha_{a}$, can take either sign depending on the sign of the scalar trilinear coupling (although note that the sign of the scalar trilinear coupling is not RG invariant). The corrections from extra matter can also be substantial, becoming very large (factor of 1.5 to 2 ) if the gauge couplings approach their semi-perturbative values.

We also showed that superoblique corrections cancel in the ratio $M_{a} / \tilde{g}_{a}^{2}$, where $\tilde{g}_{a}$ is the coupling of the gaugino to the scalar and fermionic piece of a chiral superfield (that transforms nontrivially under the associated gauge group). However, since experiments measure the gauge couplings extracted from gauge boson interactions (at least for the foreseeable future), there can be a significant correction in translating $M_{a} / \alpha_{a}$ into the true one-loop invariant $M_{a} / \tilde{g}_{a}^{2}$, if some supermultiplets are widely split in mass.

Weak scale threshold corrections proportional to a (possibly large) logarithm cancel to oneloop in the ratio $M_{a} / \alpha_{a}$. There are, however, residual finite effects when translating measured pole masses into $\overline{\mathrm{DR}}$ running masses, and effects from gaugino mixing. Thus, complete weak scale threshold corrections would be necessary for high precision. To compute the size of other effects considered in this paper, we specified the running masses at a common scale $(1 \mathrm{TeV})$ above weak scale thresholds. This was mainly for computational convenience since the complete weak scale threshold corrections can become rather complicated; of course one can always do the matching of our initial conditions to a particular weak scale model if desired.

High scale effects were also discussed. We reviewed GUT threshold corrections that have been calculated before, noting that the correction depends only on the soft breaking masses of the heavy GUT fields. GUT breaking that occurs in stages (or at a smaller scale) was also mentioned, implying the ratios $M_{a} / \alpha_{a}$ may only be applicable up to a scale that could be considerably smaller than the unification scale. Planck scale corrections from one commonly considered operator were found to cancel in the ratio $M_{a} / \alpha_{a}$, because supersymmetry enforces equal rescalings of the gaugino mass and gauge coupling squared. Finally, corrections unique to supergravity were discussed, where they were shown to depend on the form of the gauge kinetic function and the Kähler potential.

Generating gaugino masses by gauge-mediation was discussed in the final section. We calculated the one-loop gaugino masses induced by a heavy chiral multiplet, in agreement with other well-known calculations when the mass is induced entirely from gauge-mediation. Since the existence of a messenger sector and the size of the supersymmetry breaking masses is unknown, there can be arbitrarily large "corrections" to the ratio $M_{a} / \alpha_{a}$. If gauge-mediated supersym- 
metry breaking at low energy is the only source of the gaugino mass, then the two-loop effects considered in this paper are expected to be much smaller than a supergravity model, because the RG evolution is considerably reduced.

We should point out that many of the effects that we have discussed could occur simultaneously (either constructively or destructively). Determining the origin of the corrections to $M_{a} / \alpha_{a}$ would therefore be quite difficult. However, with sufficiently precise measurements of the weak scale spectrum it is expected that some of the possible disruptive effects could be ruled out. Extracting the values of $M_{a} / \alpha_{a}$ unambiguously at the high scale relies on finding techniques that can set limits or exclude the myriad effects discussed in this paper. Since these effects include corrections from e.g. extensions of the MSSM and high scale effects, an alternative point of view is that the ratios $M_{a} / \alpha_{a}$ are potentially sensitive to such physics.

Since the supersymmetric parameters $(g, M, Y, A)$ are sufficient to compute the RG evolution of $M_{a} / \alpha_{a}$, there was no need to specify any other parameters such as squark or slepton

soft masses. This allowed us to compute various corrections without any significant model dependence, although particular models were used to illustrate the effects of an extra $U(1)$. Since no attempt was made to construct complete extended models, the corrections to the MSSM ratios $M_{a} / \alpha_{a}$ should be considered as generic expectations. Some particular weak scale scenarios (or particular sets of parameters) that we have presented may be untenable when embedded in a more complete model for reasons such as vacuum stability, electroweak symmetry breaking, constraints from colliders, and constraints from cosmology. Nevertheless, unambiguous extrapolation to the high scale can be expected to be difficult regardless of the model framework, given the varied effects that impact the one-loop invariant $M_{a} / \alpha_{a}$. Careful consideration of the effects discussed in this paper would be necessary to make an unambiguous connection between weak scale and high scale physics.

\section{Acknowledgments}

I am very grateful to G.L. Kane for many discussions and encouragement that led to this work, and also to S.P. Martin and J.D. Wells for useful comments on the manuscript. I also thank M. Brhlik, H.-C. Cheng, L. Everett, C. Kolda, S.P. Martin, H. Murayama, and E. Poppitz for useful correspondence. This work was supported in part by a Rackham predoctoral fellowship and the U.S. Department of Energy.

\section{Appendix A: Relevant $\beta$-functions}

\section{A.1 Gauge and gaugino $\beta$-functions}

The $\beta$-functions for the gauge couplings, gaugino masses, and the ratio $M_{a} / \alpha_{a}$ in the $\overline{\mathrm{DR}}$ scheme [22] have been given before in Refs. [19, 20, 21, 25, 26] and we generally follow the notation 
of Ref. [25]:

$$
\begin{aligned}
\frac{d}{d t} g_{a} & =\frac{g_{a}^{3}}{16 \pi^{2}} B_{a}^{(1)}+\frac{g_{a}^{3}}{\left(16 \pi^{2}\right)^{2}}\left[\sum_{b} B_{a b}^{(2)} g_{b}^{2}-\sum_{x} C_{a}^{x} \operatorname{tr}\left(Y_{x}^{\dagger} Y_{x}\right)\right] \\
\frac{d}{d t} M_{a} & =\frac{2 g_{a}^{2}}{16 \pi^{2}} B_{a}^{(1)} M_{a}+\frac{2 g_{a}^{2}}{\left(16 \pi^{2}\right)^{2}}\left[\sum_{b} B_{a b}^{(2)} g_{b}^{2}\left(M_{a}+M_{b}\right)+\sum_{x} C_{a}^{x}\left(A_{x}-M_{a}\right) \operatorname{tr}\left(Y_{x}^{\dagger} Y_{x}\right)\right]
\end{aligned}
$$

Consequently, the two-loop RG equation for the ratio $M_{a} / \alpha_{a}$ is

$$
\frac{d}{d t} \frac{M_{a}}{g_{a}^{2}}=\frac{2}{\left(16 \pi^{2}\right)^{2}}\left[\sum_{b} B_{a b}^{(2)} g_{b}^{2} M_{b}+\sum_{x} C_{a}^{x} A_{x} \operatorname{tr}\left(Y_{x}^{\dagger} Y_{x}\right)\right]
$$

where $1 \leq a, b \leq 3$ for the MSSM $[1 \leq a, b \leq 4$ for the MSSM plus an additional $U(1)$; the $a=4$ elements of $g_{a}$ and $M_{a}$ correspond to the gauge coupling $g^{\prime}$ and gaugino mass $M^{\prime}$ associated with the $\left.U(1)^{\prime}\right]$, and $x=u, d, e(, S, D)$ for the Yukawa couplings $\mathbf{Y}_{u}, \mathbf{Y}_{d}, \mathbf{Y}_{e}\left(, Y_{S}, Y_{D}\right)$ and scalar trilinear couplings $\mathbf{A}_{u}, \mathbf{A}_{d}, \mathbf{A}_{e}\left(, A_{S}, A_{D}\right)$. (The notation $A_{x} \operatorname{tr}\left(Y_{x}^{\dagger} Y_{x}\right)$ is understood to mean $A_{x}^{i j} Y_{x}^{j i \dagger} Y_{x}^{i j}$ for the flavor indices $i, j$.) At least the first three Yukawa couplings and trilinear couplings $(u, d, e)$ are matrices in family space, however we only consider third generation couplings to be nonzero. The one-loop and two-loop coefficients are written as

$$
\begin{aligned}
B_{a}^{(1)} & =\left(b_{1}, b_{2}, b_{3}, b_{4}\right) \\
B_{a b}^{(2)} & =\left(\begin{array}{cccc}
b_{11} & b_{12} & b_{13} & b_{14} \\
b_{21} & b_{22} & b_{23} & b_{24} \\
b_{31} & b_{32} & b_{33} & b_{34} \\
b_{41} & b_{42} & b_{43} & b_{44}
\end{array}\right) \\
C_{a}^{u, d, e, S, D} & =\left(\begin{array}{ccccc}
\frac{26}{5} & \frac{14}{5} & \frac{18}{5} & \frac{6}{5} & \frac{4}{5} \\
6 & 6 & 2 & 2 & 0 \\
4 & 4 & 0 & 0 & 2 \\
c_{4 u} & c_{4 d} & c_{4 e} & c_{4 S} & c_{4 D}
\end{array}\right) .
\end{aligned}
$$

With additional matter may also come additional Yukawa couplings in the superpotential, and thus $C_{a}^{x}$ is potentially an $3 \times m$ matrix for the MSSM $[4 \times m$ matrix for the MSSM plus an additional $U(1)]$ with $m$ distinct Yukawa couplings. The coefficients $b_{a}, b_{a b}$ and $c_{4 x}$

$$
\begin{aligned}
b_{1} & =\frac{1}{10}\left(n_{Q}+8 n_{u}+2 n_{d}+3 n_{L}+6 n_{e}+3 n_{1}+3 n_{2}\right) \\
b_{2} & =\frac{1}{2}\left(3 n_{Q}+n_{L}+n_{1}+n_{2}\right)+2 n_{3}-6 \\
b_{3} & =\frac{1}{2}\left(2 n_{Q}+n_{u}+n_{d}\right)+3 n_{8}-9 \\
b_{4} & =6 n_{Q} Q_{Q}^{2}+3 n_{u} Q_{u}^{2}+3 n_{d} Q_{d}^{2}+2 n_{L} Q_{L}^{2}+n_{e} Q_{e}^{2}+2 n_{1} Q_{1}^{2}+2 n_{2} Q_{2}^{2}+n_{S} Q_{S}^{2} \\
b_{11} & =\frac{1}{150}\left(n_{Q}+128 n_{u}+8 n_{d}+27 n_{L}+216 n_{e}+27 n_{1}+27 n_{2}\right) \\
b_{12} & =\frac{3}{10}\left(n_{Q}+3 n_{L}+3 n_{1}+3 n_{2}\right) \\
b_{13} & =\frac{8}{15}\left(n_{Q}+8 n_{u}+2 n_{d}\right) \\
b_{14} & =\frac{2}{5}\left(n_{Q} Q_{Q}^{2}+8 n_{u} Q_{u}^{2}+2 n_{d} Q_{d}^{2}+3 n_{L} Q_{L}^{2}+6 n_{e} Q_{e}^{2}+3 n_{1} Q_{1}^{2}+3 n_{2} Q_{2}^{2}\right) \\
b_{21} & =\frac{1}{10}\left(n_{Q}+3 n_{L}+3 n_{1}+3 n_{2}\right)
\end{aligned}
$$




$$
\begin{aligned}
b_{22} & =\frac{7}{2}\left(3 n_{Q}+n_{L}+n_{1}+n_{2}\right)+24 n_{3}-24 \\
b_{23} & =8 n_{Q} \\
b_{24} & =2\left(3 n_{Q} Q_{Q}^{2}+n_{L} Q_{L}^{2}+n_{1} Q_{1}^{2}+n_{2} Q_{2}^{2}\right) \\
b_{31} & =\frac{1}{15}\left(n_{Q}+8 n_{u}+2 n_{d}\right) \\
b_{32} & =3 n_{Q} \\
b_{33} & =\frac{17}{3}\left(2 n_{Q}+n_{u}+n_{d}\right)+42 n_{8}-54 \\
b_{34} & =2\left(2 n_{Q} Q_{Q}^{2}+n_{u} Q_{u}^{2}+n_{d} Q_{d}^{2}\right) \\
b_{41} & =\frac{2}{5}\left(n_{Q} Q_{Q}^{2}+8 n_{u} Q_{u}^{2}+2 n_{d} Q_{d}^{2}+3 n_{L} Q_{L}^{2}+6 n_{e} Q_{e}^{2}+3 n_{1} Q_{1}^{2}+3 n_{2} Q_{2}^{2}\right) \\
b_{42} & =3\left(6 n_{Q} Q_{Q}^{2}+2 n_{L} Q_{L}^{2}+2 n_{1} Q_{1}^{2}+2 n_{2} Q_{2}^{2}\right) \\
b_{43} & =16\left(2 n_{Q} Q_{Q}^{2}+n_{u} Q_{u}^{2}+n_{d} Q_{d}^{2}\right) \\
b_{44} & =4\left(6 n_{Q} Q_{Q}^{4}+3 n_{u} Q_{u}^{4}+3 n_{d} Q_{d}^{4}+2 n_{L} Q_{L}^{4}+n_{e} Q_{e}^{4}+2 n_{1} Q_{1}^{4}+2 n_{2} Q_{2}^{4}+n_{S} Q_{S}^{4}\right) \\
c_{4 u} & =12\left(Q_{Q}^{2}+Q_{u}^{2}+Q_{2}^{2}\right) \\
c_{4 d} & =12\left(Q_{Q}^{2}+Q_{d}^{2}+Q_{1}^{2}\right) \\
c_{4 e} & =4\left(Q_{L}^{2}+Q_{e}^{2}+Q_{1}^{2}\right) \\
c_{4 s} & =4\left(Q_{S}^{2}+Q_{1}^{2}+Q_{2}^{2}\right) \\
c_{4 D} & =6\left(Q_{S}^{2}+Q_{D}^{2}+Q_{D^{c}}^{2}\right)
\end{aligned}
$$

in terms of the MSSM multiplets $\left(Q, u^{c}, d^{c}, L, e^{c}, H_{1}, H_{2}\right)$, an MSSM gauge singlet $(S)$, and the (exotic) adjoints 3 and $\mathbf{8}$. The charge assignments to $S U(3)_{c}, S U(2)_{L}, U(1)_{Y}$, and $U(1)^{\prime}$ and multiplicities $n_{i}$ of the matter supermultiplets are shown in Table 2. To incorporate the extra matter that exists in a 27 of $E_{6}$, let $n_{d} Q_{d}^{n} \rightarrow n_{d} Q_{d}^{n}+n_{D} Q_{D}^{n}+n_{D^{c}} Q_{D^{c}}^{n}$ and $n_{S} Q_{S}^{n} \rightarrow n_{S} Q_{S}^{n}+n_{\nu^{c}} Q_{\nu^{c}}^{n}$ in the above equations for $n=0,2,4$. Note that for the MSSM without a singlet, we could have ignored the distinction between $n_{1}$ and $n_{2}$, and written the above in terms of just $n_{H}=n_{1}=n_{2}$. However, the presence of the term $Y_{S} S H_{1} H_{2}$ as a substitute for $\mu H_{1} H_{2}$ in the superpotential implies $Q_{1} \neq-Q_{2}$, and thus $n_{1}$ and $n_{2}$ must be treated separately.

We illustrate the utility of the above expressions by calculating the $\beta$-function coefficients $B^{(1)}$ and $B^{(2)}$ for a few particular cases. First, for the MSSM

$$
\begin{aligned}
n_{Q} & =n_{u}=n_{d}=n_{L}=n_{e}=3 \\
n_{1} & =n_{2}=1 \\
n_{3} & =n_{8}=n_{S}=0
\end{aligned}
$$

we obtain the very well-known result

$$
\begin{aligned}
& B_{a}^{(1)}=\left(\frac{33}{5}, 1,-3\right) \\
& B_{a b}^{(2)}=\left(\begin{array}{ccc}
\frac{199}{25} & \frac{27}{5} & \frac{88}{5} \\
\frac{9}{5} & 25 & 24 \\
\frac{11}{5} & 9 & 14
\end{array}\right) .
\end{aligned}
$$




\begin{tabular}{r|ccccccccccc} 
& $Q$ & $u^{c}$ & $d^{c}$ & $L$ & $e^{c}$ & $H_{1}$ & $H_{2}$ & $D$ & $D^{c}$ & $\nu^{c}$ & $S$ \\
\hline multiplicity & $n_{Q}$ & $n_{u}$ & $n_{d}$ & $n_{L}$ & $n_{e}$ & $n_{1}$ & $n_{2}$ & $n_{D}$ & $n_{D^{c}}$ & $n_{\nu^{c}}$ & $n_{S}$ \\
\hline$S U(3)_{c}$ & $\mathbf{3}$ & $\overline{\mathbf{3}}$ & $\overline{\mathbf{3}}$ & $\mathbf{1}$ & $\mathbf{1}$ & $\mathbf{1}$ & $\mathbf{1}$ & $\mathbf{3}$ & $\overline{\mathbf{3}}$ & $\mathbf{1}$ & $\mathbf{1}$ \\
$S U(2)_{L}$ & $\mathbf{2}$ & $\mathbf{1}$ & $\mathbf{1}$ & $\mathbf{2}$ & $\mathbf{1}$ & $\mathbf{2}$ & $\mathbf{2}$ & $\mathbf{1}$ & $\mathbf{1}$ & $\mathbf{1}$ & $\mathbf{1}$ \\
$Y / 2$ of $U(1)_{Y}$ & $\frac{1}{6}$ & $-\frac{2}{3}$ & $\frac{1}{3}$ & $-\frac{1}{2}$ & 1 & $-\frac{1}{2}$ & $\frac{1}{2}$ & $-\frac{1}{3}$ & $\frac{1}{3}$ & 0 & 0 \\
$Q$ of $U(1)^{\prime}$ & $Q_{Q}$ & $Q_{u}$ & $Q_{d}$ & $Q_{L}$ & $Q_{e}$ & $Q_{1}$ & $Q_{2}$ & $Q_{D}$ & $Q_{D^{c}}$ & $Q_{\nu^{c}}$ & $Q_{S}$ \\
$2 \sqrt{15} Q_{\eta}$ of $U(1)_{\eta}$ & -2 & -2 & 1 & 1 & -2 & 1 & 4 & 4 & 1 & -5 & -5 \\
\hline
\end{tabular}

Table 2: Charge assignments for the matter considered in this paper. We did not list the 3 and 8 with multiplicities $n_{3}$ and $n_{8}$ that are in the adjoints of $S U(2)_{L}$ and $S U(3)_{c}$ respectively, since they are singlets under all other groups. The $\eta$-model of $E_{6}$ is a special case of a general $U(1)^{\prime}$ extension with the charges shown. Note that $Q_{\mathrm{em}}=T_{3}+Y / 2$, and $U(1)_{Y}$ is in the GUT normalization.

For a model with additional 5's or $\overline{\mathbf{5}}$ 's of $\mathrm{SU}(5)$ that transform as $\left(\mathbf{5}, Q_{\mathbf{5}}\right)$ and $\left(\overline{\mathbf{5}},-Q_{\mathbf{5}}\right)$ under $\left[S U(5), U(1)^{\prime}\right]$, one can compute the shift in the one-loop and two-loop $\beta$-functions

$$
\begin{aligned}
\Delta B_{a}^{(1)}= & \left(\frac{1}{2}, \frac{1}{2}, \frac{1}{2}, 5 Q_{\mathbf{5}}^{2}\right) n_{\mathbf{5}} \\
\Delta B_{a b}^{(2)}= & \left(\begin{array}{cccc}
\frac{7}{30} & \frac{9}{10} & \frac{16}{15} & 2 Q_{\mathbf{5}}^{2} \\
\frac{3}{10} & \frac{7}{2} & 0 & 2 Q_{\mathbf{5}}^{2} \\
\frac{2}{15} & 0 & \frac{17}{3} & 2 Q_{\mathbf{5}}^{2} \\
2 Q_{\mathbf{5}}^{2} & 6 Q_{\mathbf{5}}^{2} & 16 Q_{\mathbf{5}}^{2} & 20 Q_{\mathbf{5}}^{4}
\end{array}\right) n_{\mathbf{5}}
\end{aligned}
$$

and similarly for additional 10's or $\overline{\mathbf{1 0}}$ 's of $\mathrm{SU}(5)$ that transform as $\left(\mathbf{1 0}, Q_{\mathbf{1 0}}\right)$ and $\left(\overline{\mathbf{1 0}},-Q_{\mathbf{1 0}}\right)$ under $\left[S U(5), U(1)^{\prime}\right]$,

$$
\begin{aligned}
\Delta B_{a}^{(1)} & =\left(\frac{3}{2}, \frac{3}{2}, \frac{3}{2}, 10 Q_{\mathbf{1 0}}^{2}\right) n_{\mathbf{1 0}} \\
\Delta B_{a b}^{(2)} & =\left(\begin{array}{cccc}
\frac{23}{10} & \frac{3}{10} & \frac{24}{5} & 6 Q_{\mathbf{1 0}}^{2} \\
\frac{1}{10} & \frac{21}{2} & 8 & 6 Q_{\mathbf{1 0}}^{2} \\
\frac{3}{5} & 3 & 17 & 6 Q_{\mathbf{1 0}}^{2} \\
6 Q_{\mathbf{1 0}}^{2} & 18 Q_{\mathbf{1 0}}^{2} & 48 Q_{\mathbf{1 0}}^{2} & 40 Q_{\mathbf{1 0}}^{4}
\end{array}\right) n_{\mathbf{1 0}}
\end{aligned}
$$

Note that we chose the simplest assignment of the $U(1)^{\prime}$ charge, such that it is assigned vectorially and commutes with $S U(5)$. Other assignments can be handled by simply taking the appropriate components of the decomposed $\mathrm{SU}(5)$ field individually, and assigning $\mathrm{U}(1)^{\prime}$ charge as desired (for examples of other $U(1)^{\prime}$ charge assignments, see Ref. [53]). The $\left[S U(3)_{c}, S U(2)_{L}, U(1)_{Y}\right]$ pieces of the above $\beta$-functions were also calculated in e.g. Refs. 87, 28, 69, and we agree with their results.

Finally, we use the above results to compute the $\beta$-function coefficients for the $\eta$-model of $E_{6}$ with three $\mathbf{2 7}$ matter representations. The charge assignments of the $U(1)_{\eta}$ are shown in Table 2. We obtain

$$
B_{a}^{(1)}=(9,3,0,9)
$$




$$
B_{a b}^{(2)}=\left(\begin{array}{cccc}
9 & 9 & 24 & 3 \\
3 & 39 & 24 & 3 \\
3 & 9 & 48 & 3 \\
3 & 9 & 24 & 9
\end{array}\right) .
$$

The one-loop and two-loop $\beta$-function coefficients were also given in Ref. [57, 63]. We agree completely with the results in Ref. [63], and we agree with the results in Ref. [57] except for the overall sign and the values of the two-loop elements $B_{23}^{(2)}, B_{14}^{(2)}$, and $B_{41}^{(2)}$ (in our notation).

\section{A.2 Thresholds}

In running the RG equations, we will encounter two kinds of thresholds. The first, what we call "fully supersymmetric thresholds", occur when extra matter is decoupled from the spectrum in a fully supersymmetry way. The RG equations as given above are sufficient to remove matter (both the scalar and fermionic components of a chiral superfield) at any scale, provided the masses of the scalar and fermionic components are the same. Both one-loop and two-loop decoupling can be achieved; the importance of the latter for the $M_{a} / \alpha_{a}$ ratio is discussed in Sec. 3.4. The second kind of thresholds are those near the weak scale, when the supersymmetric components of the SM spectrum are decoupled. One way to treat weak scale thresholds is to decouple the supersymmetric components from the $\beta$-functions, creating non-supersymmetric effective theories. Expressions for the corrections to the one-loop $\beta$-functions are given in Ref. [《], and we have translated their results into the formalism we have used above:

$$
\begin{aligned}
n_{Q} & \rightarrow \frac{1}{3} n_{u}^{(f)}+\frac{1}{3} n_{d}^{(f)}+\frac{1}{6} n_{\tilde{u}_{L}}^{(s)}+\frac{1}{6} n_{\tilde{d}_{L}}^{(s)} \\
n_{u} & \rightarrow \frac{2}{3} n_{u}^{(f)}+\frac{1}{3} n_{\tilde{u}_{R}}^{(s)} \\
n_{d} & \rightarrow \frac{2}{3} n_{d}^{(f)}+\frac{1}{3} n_{\tilde{d}_{R}}^{(s)} \\
n_{L} & \rightarrow \frac{1}{3} n_{v}^{(f)}+\frac{1}{3} n_{e}^{(f)}+\frac{1}{6} n_{\tilde{v}}^{(s)}+\frac{1}{6} n_{\tilde{e}_{L}}^{(s)} \\
n_{e} & \rightarrow \frac{2}{3} n_{e}^{(f)}+\frac{1}{3} n_{\tilde{e}_{R}}^{(s)} \\
n_{1} & \rightarrow \frac{2}{3} n_{\tilde{H}_{1}}^{(f)}+\frac{1}{3} n_{H_{1}}^{(s)} \\
n_{2} & \rightarrow \frac{2}{3} n_{\tilde{H}_{2}}^{(f)}+\frac{1}{3} n_{H_{2}}^{(s)}
\end{aligned}
$$

where $f$ is the fermion and $s$ is the complex scalar. In addition, the $\beta$-function coefficients for the non-Abelian gauge couplings have the further changes

$$
\begin{array}{llll}
b_{2}: & -6 & \rightarrow & -\frac{22}{3}+\frac{4}{3} \theta_{\tilde{W}} \\
b_{3}: & -9 & \rightarrow & -11+2 \theta_{\tilde{g}}
\end{array}
$$

where $\theta_{\tilde{W}, \tilde{g}}=0$ or 1 if the renormalization scale $Q$ is less than or greater than $\left|M_{\tilde{W}, \tilde{g}}\right|$ respectively. Finally, note that the two-loop corrections due to weak scale thresholds could in principle be calculated, but we ignore these corrections since they are not enhanced by a large logarithm (unlike fully supersymmetric thresholds), see Ref. [37] for further discussion. 


\section{A.3 Yukawa and scalar trilinear $\beta$-functions}

The general form of the one-loop RG equations for the Yukawa couplings and soft scalar trilinear couplings is

$$
\begin{aligned}
\frac{d}{d t} Y_{i} & =\frac{Y_{i}}{16 \pi^{2}}\left[\sum_{j} c_{i j} Y_{j}^{2}-\sum_{k} d_{i k} g_{k}^{2}\right] \\
\frac{d}{d t} A_{i} & =\frac{1}{8 \pi^{2}}\left[\sum_{j} c_{i j} Y_{j}^{2} A_{j}+\sum_{k} d_{i k} g_{k}^{2} M_{k}\right]
\end{aligned}
$$

where the coefficients are

$$
\begin{aligned}
c_{i j}= & \left(\begin{array}{ccccc}
6 & 1 & 0 & 1 & 0 \\
1 & 6 & 1 & 1 & 0 \\
0 & 3 & 4 & 1 & 0 \\
3 & 3 & 1 & 4 & 3 \\
0 & 0 & 0 & 2 & 5
\end{array}\right) \\
d_{i k}= & \left(\begin{array}{cccc}
\frac{13}{15} & 3 & \frac{16}{3} & 2\left(Q_{Q}^{2}+Q_{u}^{2}+Q_{2}^{2}\right) \\
\frac{7}{15} & 3 & \frac{16}{3} & 2\left(Q_{Q}^{2}+Q_{d}^{2}+Q_{1}^{2}\right) \\
\frac{9}{5} & 3 & 0 & 2\left(Q_{L}^{2}+Q_{e}^{2}+Q_{1}^{2}\right) \\
\frac{3}{5} & 3 & 0 & 2\left(Q_{S}^{2}+Q_{1}^{2}+Q_{2}^{2}\right) \\
\frac{2}{15} & 0 & \frac{16}{3} & 2\left(Q_{S}^{2}+Q_{D}^{2}+Q_{D^{c}}^{2}\right)
\end{array}\right)
\end{aligned}
$$

for $i, j=(u, d, e, S, D)$ and $k=1,2,3,4$. We are neglecting the first and second generation Yukawa couplings and associated mixings between generations. Two-loop corrections to the Yukawa couplings [4, 8, 25, 26] and the associated scalar trilinear couplings [25, 26] have been calculated, and expressions for the two-loop corrections to $Y_{S}$ and $Y_{D}$ could be easily calculated from Refs. [25, 26]. We consistently neglect two-loop corrections to Yukawa couplings since they are of the same order as three-loop corrections (which we also neglect) to the main observables $g_{a}, M_{a}$, and $M_{a} / \alpha_{a}$ relevant to this paper.

\section{Appendix B: Cancellation of $U(1)^{\prime}$ anomalies}

\section{B.1 General conditions}

There are six anomalies associated with enlarging the MSSM gauge group to include an extra $U(1)^{\prime}$ that need to be canceled if we expect a sensible low energy effective theory. These are the mixed gauge anomalies, $\left[S U(3)_{c}\right]^{2} U(1)^{\prime},\left[S U(2)_{L}\right]^{2} U(1)^{\prime},\left[U(1)_{Y}\right]^{2} U(1)^{\prime},\left[U(1)^{\prime}\right]^{2} U(1)_{Y}$, the triangle anomaly $\left[U(1)^{\prime}\right]^{3}$, and the mixed gravitational anomaly $U(1)^{\prime}$ [gravity $]^{2}$. With the addition of the mixed gravitational anomaly, this appendix follows closely the discussion of $U(1)^{\prime}$ anomalies in Ref. [49].

With the particle content, multiplicities, and $U(1)^{\prime}$ charges stated in Table 2, the cancellation of the $\left[S U(3)_{c}\right]^{2} U(1)^{\prime},\left[S U(2)_{L}\right]^{2} U(1)^{\prime},\left[U(1)_{Y}\right]^{2} U(1)^{\prime},\left[U(1)^{\prime}\right]^{2} U(1)_{Y},\left[U(1)^{\prime}\right]^{3}, U(1)^{\prime}[\text { gravity }]^{2}$ 
anomalies require, respectively,

$$
\begin{array}{rl}
0= & 2 n_{Q} Q_{Q}+n_{u} Q_{u}+n_{d} Q_{d}+n_{D} Q_{D}+n_{D^{c}} Q_{D^{c}} \\
0= & 3 n_{Q} Q_{Q}+n_{L} Q_{L}+n_{1} Q_{1}+n_{2} Q_{2} \\
0= & 2 n_{Q} Q_{Q}+16 n_{u} Q_{u}+4 n_{d} Q_{d}+6 n_{L} Q_{L}+12 n_{e} Q_{e}+6 n_{1} Q_{1}+6 n_{2} Q_{2}+4 n_{D} Q_{D}+4 n_{D^{c}} Q_{D^{c}} \\
0= & n_{Q} Q_{Q}^{2}-2 n_{u} Q_{u}^{2}+n_{d} Q_{d}^{2}-n_{L} Q_{L}^{2}+n_{e} Q_{e}^{2}-n_{1} Q_{1}^{2}+n_{2} Q_{2}^{2}-n_{D} Q_{D}^{2}+n_{D^{c}} Q_{D^{c}}^{2} \\
0= & 6 n_{Q} Q_{Q}^{3}+3 n_{u} Q_{u}^{3}+3 n_{d} Q_{d}^{3}+2 n_{L} Q_{L}^{3}+n_{e} Q_{e}^{3}+2 n_{1} Q_{1}^{3}+2 n_{2} Q_{2}^{3}+3 n_{D} Q_{D}^{3}+3 n_{D^{c}} Q_{D^{c}}^{3} \\
& +n_{S} Q_{S}^{3}+n_{\nu^{c}} Q_{\nu^{c}}^{3} \\
0 & 6 n_{Q} Q_{Q}+3 n_{u} Q_{u}+3 n_{d} Q_{d}+2 n_{L} Q_{L}+n_{e} Q_{e}+2 n_{1} Q_{1}+2 n_{2} Q_{2}+3 n_{D} Q_{D}+3 n_{D^{c}} Q_{D^{c}} \\
& +n_{S} Q_{S}+n_{\nu^{c}} Q_{\nu^{c}} .
\end{array}
$$

For completeness, the further constraint of preventing radiatively generated kinetic mixing $\operatorname{tr}(Y Q)=$ 0 is

$$
0=n_{Q} Q_{Q}-2 n_{u} Q_{u}+n_{d} Q_{d}-n_{L} Q_{L}+n_{e} Q_{e}-n_{1} Q_{1}+n_{2} Q_{2}-n_{D} Q_{D}+n_{D^{c}} Q_{D^{c}} .
$$

\section{B.2 Minimal $U(1)^{\prime}$ model}

The model of Ref. [49] has the superpotential

$$
W=Y_{t} Q H_{2} u^{c}+Y_{S} S H_{1} H_{2},
$$

(devoid of Yukawa couplings to the $b$ or $\tau$ sector), with the MSSM spectrum and one singlet $S$, and the MSSM group plus an additional $U(1)^{\prime}$. The authors of Ref. 449] wrote down one simple example of anomaly cancellation in their model,

$$
\begin{aligned}
Q_{Q} & =-\frac{1}{3} Q_{1} \\
Q_{u} & =\frac{1}{3}\left(Q_{1}-3 Q_{2}\right) \\
Q_{d} & =\frac{1}{3}\left(Q_{1}+3 Q_{2}\right) \\
Q_{L} & =-Q_{2} \\
Q_{e} & =Q_{2}-Q_{1} \\
Q_{S} & =-\left(Q_{1}+Q_{2}\right),
\end{aligned}
$$

where the charges given above for $(Q, u, d, L, e)$ are assigned only to the third generation (first and second generations have zero $U(1)^{\prime}$ charge). To prevent radiatively generated kinetic mixing, we also impose the constraint

$$
Q_{2}=\frac{4}{9} Q_{1} .
$$

Thus all of the charges are determined up to one "overall" charge, which we take to be $Q_{1}$. Since the gauge coupling $g^{\prime}$ is always accompanied one power of some $U(1)^{\prime}$ charge, we can therefore choose to rescale either $Q_{1}$ or $g^{\prime}$, without loss of generality. If the $U(1)^{\prime}$ were embedded in a higher rank group at the high scale, then the rescaling factor would be fixed by the particular embedding. 
Note that experimental bounds on $Z-Z^{\prime}$ mixing also impose constraints on the size of $g^{\prime} Q_{1}$, and may not even allow Eq. (54) to be satisfied in the particular scenarios where the vev of $S$ is comparable to the vevs $v_{1}$ and $v_{2}$ (from $H_{1}$ and $H_{2}$ ) [49]. The mixing angle in the $Z-Z^{\prime}$ system is given by

$$
\tan 2 \alpha_{Z-Z^{\prime}}=\frac{g^{\prime} \sqrt{g_{1}^{2}+g_{2}^{2}}\left(v_{1}^{2} Q_{1}-v_{2}^{2} Q_{2}\right)}{M_{Z^{\prime}}^{2}-M_{Z}^{2}} .
$$

We find the intriguing result that simultaneously satisfying Eq. (54), and zero mixing occurs when $\tan \beta=\frac{3}{2}$. Of course the mixing could be nonzero but simply suppressed by a large $Z^{\prime}$ mass, although generally $Q_{1} Q_{2}>0$ is needed for a partial cancellation in the numerator of the above expression [which is compatible with Eq. (54)]. In any case, for our purposes we will take Eq. (54) without specifying $\tan \beta$, and assume that appropriate (larger) vevs of $S$ are induced as necessary to avoid the experimental bounds.

\section{B.3 $\quad E_{6}$ model}

Using the charge assignments given in Table 2, it is easy to show that the $\eta$-model of $E_{6}$ with three generations of $\mathbf{2 7}$ s cancels all of the anomalies above. This is not at all surprising, since it is well known that theories with matter in complete representations of a non-Abelian group are nonanomalous. Three generations of $\mathbf{2 7} \mathrm{s}$ are sufficient to incorporate the matter content of the MSSM, but also include two extra generations of up-type and down-type Higgs doublets, three generations of right-handed neutrinos, and three generations of MSSM gauge singlets.

\section{References}

[1] For more theoretically oriented reviews, see for example H.P. Nilles, Phys. Rept. 110, 1 (1984); J.D. Lykken, [hep-th/9612114] (TASI-96 lectures); M. Dine, [hep-ph/9612389] (TASI-96 lectures); K.R. Dienes, C. Kolda, [hep-ph/9712322] (to appear in "Perspectives on Supersymmetry", World Scientific, Editor G.L. Kane).

For more phenomenologically oriented reviews, see for example H.E. Haber, G.L. Kane, Phys. Rept. 117, 75 (1985); H. Baer, et al., [hep-ph/9503479] (contribution to the DPF working group on "Electroweak Symmetry Breaking and Beyond the Standard Model"); X. Tata, [hep-ph/9706307] (lectures given at IX Jorge A. Swieca Summer School, 1997); S.P. Martin, [hep-ph/9709356] (to appear in "Perspectives on Supersymmetry", World Scientific, Editor G.L. Kane); S. Dawson, [hep-ph/9712464] (TASI-97 lectures).

[2] G.G. Ross, R.G. Roberts, Nucl. Phys. B377, 571 (1992).

[3] J. Hisano, H. Murayama, T. Yanagida, Phys. Rev. Lett. 69, 1014 (1992).

[4] V. Barger, M.S. Berger, P. Ohmann, Phys. Rev. D47, 1093 (1993) [hep-ph/9209232].

[5] P. Langacker, N. Polonsky, Phys. Rev. D47, 4028 (1993) [hep-ph/9210235]. 
[6] M. Carena, S. Pokorski, C.E.M. Wagner, Nucl. Phys. B406, 59 (1993) [hep-ph/9303202].

[7] M. Olechowski, S. Pokorski, Nucl. Phys. B404, 590 (1993) [hep-ph/9303274].

[8] D.J. Castaño, E.J. Piard, P. Ramond, Phys. Rev. D49, 4882 (1994) [hep-ph/9308335].

[9] V. Barger, M.S. Berger, P. Ohmann, Phys. Rev. D49, 4908 (1994) [hep-ph/9311269].

[10] G.L. Kane, C. Kolda, L. Roszkowski, J.D. Wells, Phys. Rev. D49, 6173 (1994) [hep-ph/9312272].

[11] J.L. Lopez, D.V. Nanopoulos, A. Zichichi, Prog. Part. Nucl. Phys. 33, 303 (1994) [hep-ph/9402299].

[12] P.H. Chankowski, Z. Pluciennik, S. Pokorski, Nucl. Phys. B439, 23 (1995) [hep-ph/9411233].

[13] K. Inoue, A. Kakuto, H. Komatsu, S. Takeshita, Prog. Theor. Phys. 68, 927 (1982); ibid., 71, 413 (1984); L.E. Ibáñez, Nucl. Phys. B218, 514 (1983); L.E. Ibáñez, C. López, Phys. Lett. B 126, 54 (1983); ibid., Nucl. Phys. B233, 511 (1984); L. Alvarez-Gaume, J. Polchinski, M.B. Wise, Nucl. Phys. B211, 495 (1983).

[14] See R.N. Mohapatra, Unification and Supersymmetry (Springer-Verlag, New York, 1992); R.N. Mohapatra, [hep-ph/9801235] (TASI-97 lectures), and references therein.

[15] See for example Nilles in Ref. [1], and also Ref. 82] for more detailed calculations in particular stringy scenarios.

[16] A. Zichichi, Lecture given at the XXXII Course of "Ettore Majorana" International School of Subnuclear Physics, 1994; see also Ref. [11].

[17] M. Carena, P. Chankowski, M. Olechowski, S. Pokorski, C.E.M. Wagner, Nucl. Phys. B491, 103 (1997) [hep-ph/9612261].

[18] G.L. Kane, [hep-ph/9709318] (talk given at the Fifth International Conference on Supersymmetries in Physics, 1997).

[19] Y. Yamada, Phys. Lett. B 316, 109 (1993) [hep-ph/9307217].

[20] S.P. Martin, M.T. Vaughn, Phys. Lett. B 318, 331 (1993) [hep-ph/9308222].

[21] Y. Yamada, Phys. Rev. Lett. 72, 25 (1994) [hep-ph/9308304].

[22] W. Siegel, Phys. Lett. B 84, 193 (1979); D.M. Capper, D.R.T. Jones, P. van Nieuwenhuizen, Nucl. Phys. B167, 479 (1980); I. Jack, D.R.T. Jones, S.P. Martin, M.T. Vaughn, Y. Yamada Phys. Rev. D50, 5481 (1994) [hep-ph/9407291].

[23] P.M. Ferreira, I. Jack, D.R.T. Jones, Phys. Lett. B 387, 80 (1996) [hep-ph/9605440]; I. Jack, D.R.T. Jones, C.G. North, Phys. Lett. B 386, 138 (1996) [hep-ph/9606323].

[24] I. Jack, D.R.T. Jones, Phys. Lett. B 415, 383 (1997) [hep-ph/9709364]; L.V. Avdeev, D.I. Kazakov, I.N. Kondrashuk, Nucl. Phys. B510, 289 (1998) [hep-ph/9709397]. 
[25] S.P. Martin, M. Vaughn, Phys. Rev. D50, 2282 (1994) [hep-ph/9311340].

[26] Y. Yamada, Phys. Rev. D50, 3537 (1994) [hep-ph/9401241].

[27] For a recent review, see I. Jack, D.R.T. Jones, [hep-ph/9707278] (to appear in "Perspectives on Supersymmetry", World Scientific, Editor G.L. Kane).

[28] C.F. Kolda, J. March-Russell, Phys. Rev. D55, 4252 (1997) [hep-ph/9609480].

[29] V.A. Novikov, M.A. Shifman, A.I. Vaishtein, V.I. Zakharov, Nucl. Phys. B229, 381 (1983); ibid., Nucl. Phys. B260, 157 (1985); ibid., Phys. Lett. B 166, 334 (1986); M. Shifman, Int. J. Mod. Phys. A11 (1996) 5761 [hep-ph/9606281]; N. Arkani-Hamed, H. Murayama, [hep-th/9707133].

[30] J. Hisano, M. Shifman, Phys. Rev. D56, 5475 (1997) [hep-ph/9705417];

[31] S. Ambrosanio, G.L. Kane, G.D. Kribs, S.P. Martin, S. Mrenna, Phys. Rev. Lett. 76, 3498 (1996) [hep-ph/9602239]; ibid., Phys. Rev. D55, 1372 (1997) [hep-ph/9607414].

[32] N. Arkani-Hamed, H.-C. Cheng, T. Moroi, Phys. Lett. B 387, 529 (1996) [hep-ph/9607463] .

[33] See for example G.R. Farrar, [hep-ph/9710277] (talk given at Fifth International Conference on Supersymmetries in Physics, 1997), and references therein.

[34] D. Pierce, A. Papadopoulos, Nucl. Phys. B430, 278 (1994) [hep-ph/9403240].

[35] J. Bagger, K. Matchev, D. Pierce, Phys. Lett. B 348, 443 (1995) [hep-ph/9501277].

[36] D.M. Pierce, J.A. Bagger, K. Matchev, R. Zhang, Nucl. Phys. B491, 3 (1997) [hep-ph/9606211].

[37] L. Hall, Nucl. Phys. B178, 75 (1981).

[38] J.L. Feng, H. Murayama, M.E. Peskin, X. Tata, Phys. Rev. D52, 1418 (1995) [hep-ph/9502260].

[39] M.M. Nojiri, K. Fujii, T. Tsukamoto, Phys. Rev. D54, 6756 (1996) [hep-ph/9606370].

[40] K. Hikasa, Y. Nakamura, Z. Phys. C 70, 139 (1996) [hep-ph/9501382]; erratum, Z. Phys. C 71, 356 (1996).

[41] H.-C. Cheng, J.L. Feng, N. Polonsky, Phys. Rev. D56, 6875 (1997) [hep-ph/9706438].

[42] L. Randall, [hep-ph/9706478] (talk given at the Fifth International Conference on Supersymmetries in Physics, 1997).

[43] M.M. Nojiri, D.M. Pierce, Y. Yamada, Phys. Rev. D57, 1539 (1998) [hep-ph/9707244];

[44] E. Katz, L. Randall, S. Su, [hep-ph/9801416].

[45] S. Dimopoulos, G.F. Giudice, Phys. Lett. B 357, 573 (1995) [hep-ph/9507282]. 
[46] G. Dvali, A. Pomarol, Phys. Rev. Lett. 77, 3728 (1996) [hep-ph/9607383]; R.N. Mohapatra, A. Riotto, Phys. Rev. D55, 1138 (1997) [hep-ph/9608441]; R.-J. Zhang, Phys. Lett. B 402, 101 (1997) [hep-ph/9702333].

[47] A.G. Cohen, D.B. Kaplan, A.E. Nelson, Phys. Lett. B 388, 588 (1996) [hep-ph/9607394]; A.G. Cohen, D.B. Kaplan, F. Lepeintre, A.E. Nelson Phys. Rev. Lett. 78, 2300 (1997) [hep-ph/9610252]; A.E. Nelson, D. Wright Phys. Rev. D56, 1598 (1997) [hep-ph/9702359]; S. Ambrosanio, A.E. Nelson, Phys. Lett. B 411, 283 (1997) [hep-ph/9707242].

[48] For a review, see M. Cvetič, P. Langacker, [hep-ph/9707451] (to appear in "Perspectives on Supersymmetry", World Scientific, Editor G.L. Kane), and references therein.

[49] M. Cvetič, D.A. Demir, J.R. Espinosa, L. Everett, P. Langacker, Phys. Rev. D56, 2861 (1997) [hep-ph/9703317]; erratum, to be published.

[50] See for example S.F. King, P.L. White, Phys. Rev. D52, 4183 (1995) [hep-ph/9505326] and references therein.

[51] B. Holdom, Phys. Lett. B 166, 196 (1986).

[52] F. del Aguila, M. Quirós, F. Zwirner, Nucl. Phys. B287, 419 (1987); F. del Aguila, G.D. Coughlan, M. Quirós, Nucl. Phys. B307, 633 (1988); F. del Aguila, M. Masip, M. Pérez-Victoria, Nucl. Phys. B456, 531 (1995) [hep-ph/9507455].

[53] K.S. Babu, C. Kolda, J. March-Russell, Phys. Rev. D54, 4635 (1996) [hep-ph/9603212].

[54] K.R. Dienes, C. Kolda, J. March-Russell, Nucl. Phys. B492, 104 (1997) [hep-ph/9610479].

[55] K.S. Babu, C. Kolda, J. March-Russell, [hep-ph/9710441].

[56] P. Binétruy, S. Dawson, I. Hinchliffe, M. Sher, Nucl. Phys. B273, 501 (1986).

[57] J. Ellis, K. Enqvist, D.V. Nanopoulos, F. Zwirner, Nucl. Phys. B276, 14 (1986).

[58] L.E. Ibáñez, J. Mas, Nucl. Phys. B286, 107 (1987).

[59] F. del Aguila, J.A. González, M. Quirós, Nucl. Phys. B307, 571 (1988).

[60] For a review, see J.L. Hewett, T.G. Rizzo, Phys. Rept. 183, 193 (1989).

[61] K.R. Dienes, Phys. Rept. 287, 447 (1997) [hep-th/9602045].

[62] J. Ellis, D.V. Nanopoulos, S.T. Petcov, F. Zwirner, Nucl. Phys. B283, 93 (1987).

[63] A.J. Davies, R.R. Volkas, G.C. Joshi, Phys. Rev. D37, 1292 (1988).

[64] E. Keith, E. Ma, Phys. Rev. D54, 3587 (1996) [hep-ph/9603353].

[65] M. Dine, A.E. Nelson, Phys. Rev. D48, 1277 (1993) [hep-ph/9303230]; M. Dine, A.E. Nelson, Y. Shirman, Phys. Rev. D51, 1362 (1995) [hep-ph/9408384]; M. Dine, A.E. Nelson, Y. Nir, Y. Shirman, Phys. Rev. D53, 2658 (1996) [hep-ph/9507378]. 
[66] For a recent comprehensive review with extensive references, see G.F. Giudice, R. Rattazzi, [hep-ph/9801271].

[67] S.P. Martin, P. Ramond, Phys. Rev. D51, 6515 (1995) [hep-ph/9501244].

[68] S.P. Martin, Phys. Rev. D55, 3177 (1997) [hep-ph/9608224].

[69] D. Ghilencea, M. Lanzagorta, G.G. Ross, Nucl. Phys. B511, 3 (1998) [hep-ph/9707401].

[70] D. Ghilencea, M. Lanzagorta, G.G. Ross, Phys. Lett. B 415, 253 (1997) [hep-ph/9707462].

[71] J. Hisano, H. Murayama, T. Goto, Phys. Rev. D49, 1446 (1994).

[72] The sign in front of the $1 / 4 \pi$ is consistent with Eq. (10), but opposite to that in Eq. (15) of Ref. [71]. The sign is consistent with an equivalent expression given in Ref. [28].

[73] Y. Kawamura, H. Murayama, M. Yamaguchi, Phys. Lett. B 324, 52 (1994) [hep-ph/9402254].

[74] C.T. Hill, Phys. Lett. B 135, 47 (1984).

[75] Q. Shafi, C. Wetterich, Phys. Rev. Lett. 52, 875 (1984).

[76] L.J. Hall, U. Sarid, Phys. Rev. Lett. 70, 2673 (1993) [hep-ph/9210240].

[77] D. Ring, S. Urano, R. Arnowitt, Phys. Rev. D52, 6623 (1995) [hep-ph/9501247].

[78] T. Dasgupta, P. Mamales, P. Nath, Phys. Rev. D52, 5366 (1995) [hep-ph/9501325].

[79] J. Ellis, K. Enqvist, D.V. Nanopoulos, K. Tamvakis, Phys. Lett. B 155, 381 (1985).

[80] M. Drees, Phys. Lett. B 158, 409 (1985); M. Drees, Phys. Rev. D33, 1468 (1986).

[81] E. Cremmer, S. Ferrara, L. Girardello, A. van Proeyen, Nucl. Phys. B212, 413 (1983).

[82] A. Font, L.E. Ibáñez, D. Lüst, F. Quevedo, Phys. Lett. B 245, 401 (1990); L.E. Ibáñez, D. Lüst, Nucl. Phys. B382, 305 (1992) [hep-th/9202046]; B. de Carlos, J.A. Casas, C. Muñoz, Phys. Lett. B 299, 234 (1993) [hep-ph/9211266]; V.S. Kaplunovsky, J. Louis, Phys. Lett. B 306, 269 (1993) [hep-th/9303040]; A. Brignole, L.E. Ibáñez, C. Muñoz, Nucl. Phys. B422, 125 (1994) [hep-ph/9308271].

[83] E. Poppitz, S.P. Trivedi, Phys. Lett. B 401, 38 (1997) [hep-ph/9703246].

[84] M. Picariello, A. Strumia, [hep-ph/9802446].

[85] S. Dimopoulos, M. Dine, S. Raby, S. Thomas, Phys. Rev. Lett. 76, 3494 (1996) [hep-ph/9601367].

[86] S. Ambrosanio, G.L. Kane, G.D. Kribs, S.P. Martin, S. Mrenna, Phys. Rev. D54, 5395 (1996) [hep-ph/9605398]; S. Ambrosanio, G.D. Kribs, S.P. Martin, Phys. Rev. D56, 1761 (1997) [hep-ph/9703211].

[87] C.D. Carone, H. Murayama, Phys. Rev. D53, 1658 (1996) [hep-ph/9510219]. 